\title{
All-microwave nonadiabatic multiqubit geometric phase gate for superconducting qubits
}

\author{
T.-Q. Cai $\odot,{ }^{*}$ J.-H. Wang $\odot,{ }^{*}$ Z.-L. Wang, X.-Y. Han, Y.-K. Wu $\odot,{ }^{\dagger}$ Y.-P. Song $\odot, ‡$ and L.-M. Duan ${ }^{\S}$ \\ Center for Quantum Information, Institute for Interdisciplinary Information Sciences, Tsinghua University, Beijing 100084, China
}

(Received 14 January 2021; revised 27 August 2021; accepted 13 October 2021; published 26 October 2021)

\begin{abstract}
Geometric phase gates are promising tools toward robust quantum computing owing to their robustness against certain control errors and decoherence. Here, we propose a multiqubit architecture with a nonadiabatic geometric phase gate scheme that is feasible in widely used superconducting qubit designs such as transmon and fluxonium. Through segmented microwave drive on multiple qubits coupled off-resonantly to a common resonator, the geometric phase gate is obtained from the qubit-state-dependent displacement of the resonator without finetuning the qubit frequencies. Fidelity above $99.99 \%$ is achieved in simulation under the available experimental parameters. Our scheme uses all-microwave control and only exploits the lowest qubit levels with long coherence time; thus it is desirable for experiments. Together with the single-qubit holonomic gates demonstrated in earlier experiments, our scheme can realize universal all-geometric quantum computing, and it also finds applications in quantum simulation with many-body interactions.
\end{abstract}

DOI: 10.1103/PhysRevResearch.3.043071

\section{INTRODUCTION}

When a quantum system undergoes a cyclic evolution, it acquires a geometric phase, or more generally is subjected to a unitary operator known as a holonomy, which solely depends on the path geometry but not on how fast it is traversed [1-4]. Since its discovery, geometric phases have found wide applications [5] such as the topological effects in condensed matter systems [6] and quantum simulation of gauge field theories [7]. Furthermore, owing to the intrinsic robustness against certain types of control errors [8-11] and decoherence $[10,12,13]$, geometric gates make a preferable approach toward fault-tolerant quantum computing, namely, the idea of geometric or holonomic quantum computing [14-17].

Geometric single-qubit and two-qubit gates have been demonstrated in experiments in various systems such as nuclear magnetic resonance (NMR) [18-20], nitrogen vacancy (NV) centers [21-25], trapped ions [26,27], and superconducting circuits [28-34]. In a superconducting qubit system, which is one of the leading platforms for quantum computing, experimental demonstrations of geometric gates are generally in the nonadiabatic regime, where careful cancellation of the dynamical effects is needed [35-38]. In comparison, the adiabatic ones require longer gate time and are hence more vulnerable to decoherence in the system, despite being

\footnotetext{
* These authors contributed equally to this work.

†wyukai@mail.tsinghua.edu.cn

†ypsong@mail.tsinghua.edu.cn

${ }^{\S}$ Imduan@tsinghua.edu.cn
}

Published by the American Physical Society under the terms of the Creative Commons Attribution 4.0 International license. Further distribution of this work must maintain attribution to the author(s) and the published article's title, journal citation, and DOI. more robust against noise in control parameters [39]. Another possible direction is the unconventional geometric two-qubit gate [40], where the dynamical phase is shown to be always proportional to the geometric ones. Thus the delicate cancellation of the dynamical phase is no longer needed even in the nonadiabatic case, which allows strong noise resilience $[13,40]$. Actually, the same mechanism was proposed earlier in ion traps without being recognized as a geometric gate [41-43] and has shown high gate fidelity [26,44,45].

In the aforementioned experimental realization of geometric gates in superconducting circuits, usually the higher excited levels of the circuit are exploited to encode the qubit states or to assist the gate implementation [28-32,34]. These schemes are thus subjected to more severe decoherence than that for the lowest two qubit levels. Proposals using only the low qubit levels exist based on the unconventional geometric phase through the coupling to a resonator [46,47]; however, they require specially designed superconducting qubits as well as time-dependent flux control for the gate operation and hence have not been realized yet. Similar ideas have also been used in resonator-induced phase gates [46,48-51], where the microwave control is applied on the shared resonator mode for a global entangling gate. If gates among selected qubits are desired, one will need to tune the frequency of each qubit to and away from resonance on demand [33] or use complicated spin echo pulses [47].

Here, we propose a multiqubit architecture with an allmicrowave scheme for multiqubit geometric phase gates which is compatible with popular superconducting qubit designs such as transmon and fluxonium. An all-microwave control scheme can provide frequency selectivity and allows us to use fixed-frequency computational qubits, thereby minimizing the sensitivity of the qubits with respect to the sources of possible noise. Moreover, by applying microwave drive among multiple qubits off-resonantly coupled to a common resonator, we generate qubit-state-dependent rotation 
and displacement on the cavity mode, thus obtaining the unconventional geometric phase between the desired qubits [40]. The residual entanglement between the qubit states and the resonator is suppressed by segmented microwave control, and fidelity above $99.99 \%$ is achieved in the numerical simulation using realistic parameters. A similar control scheme based on segmented laser pulses has likewise been used for design of high-fidelity trapped-ion quantum gates [52]. A well-known example of the all-microwave gate scheme is the cross-resonance $(\mathrm{CR})$ gate, which has been experimentally realized with gate fidelity reaching 99\% [53]. However, compared with the $\mathrm{CR}$ gate, our scheme by using the single microwave drive tone on the fixed-frequency computational qubits is beneficial to improving the on-off ratio of the coupling between the qubits and further decreasing frequency crowding in a large superconducting network. Furthermore, compared with earlier schemes of geometric gates for superconducting qubits [29,32-34,51,54,55], our scheme uses only the lowest qubit levels and the cavity mode without touching higher-energy states, and thus provides relatively long coherence time; also, the single-tone microwave strategy on fixed-frequency qubits further reduces the circuit complexity to prevent extra decoherence. Moreover, the proposed scheme allows for one-step implementation of a multiqubit entangling state with the global geometric phase gate by optimizing the segmented driving pulses. Together with the single-qubit geometric gates demonstrated in previous experiments [32], universal all-geometric quantum computing can thus be realized. The multiqubit entangling gate implemented here can also be applied in quantum simulation with many-body interaction Hamiltonian.

This paper is organized as follows. In Sec. II, we introduce a system Hamiltonian for a two-qubit geometric phase gate under an ideal two-level qubit model. In Sec. III, we analyze the two-qubit gate scheme in a multilevel transmon model, treating the transmon as a Duffing oscillator. We show that the presence of the high energy level can be effectively optimized with the multisegment driving scheme. Section IV extends to a global geometric phase gate scheme for one-step implementation of multiqubit entangling state by optimizing the segmented driving pulses.

\section{TWO-QUBIT GEOMETRIC PHASE GATE IN A QUBIT MODEL}

\section{A. Model and single-segment scheme}

We consider a generic system of $n$ superconducting qubits $\left(Q_{1}, Q_{2}, \ldots, Q_{n}\right)$ and a microwave resonator (bus), as outlined in Fig. 1(a). Suppose we want to implement a two-qubit gate between $Q_{1}$ and $Q_{2}$ as shown in Fig. 1(b). Here, we first describe the gate scheme under the two-level approximation of the qubits, and later we generalize it to include the effects of higher levels. The two qubits are coupled to the bus, modeled as a single-mode harmonic oscillator, at the coupling strength $g_{i}(i=1,2)$, and are each driven via an XY control line by a microwave tone with an amplitude $\Omega_{i}(i=1,2)$ and a frequency $\omega_{d}$. For clarity, we consider a system which satisfies the rotating-wave approximation, and the situation without the rotating-wave approximation will be discussed later. The full (a)

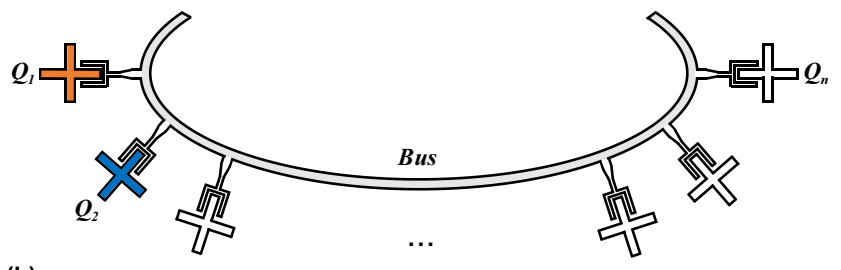

(b)

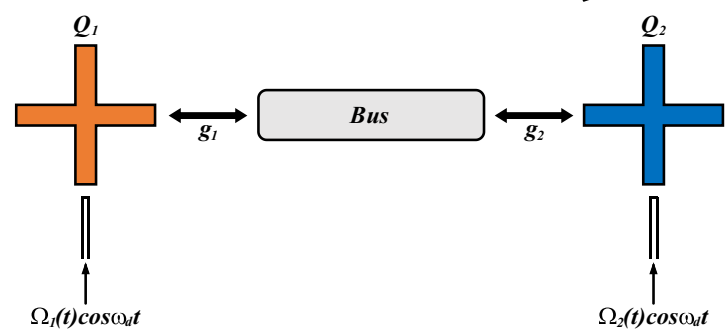

FIG. 1. (a) The system consists of $n$ superconducting qubits $\left(Q_{1}, Q_{2}, \ldots, Q_{n}\right)$ coupled to a common microwave resonator (bus). (b) A schematic of the two-qubit geometric phase gate. The two target qubits are selectively driven by a microwave pulse $\Omega_{i}(t) \cos \omega_{d} t$ $(i=1,2)$ via the XY control line.

system Hamiltonian under the rotating-wave approximation can be expressed as

$$
\begin{aligned}
H= & -\sum_{i=1,2} \frac{\omega_{i}}{2} \sigma_{z}^{i}+\omega_{b} a^{\dagger} a+\sum_{i=1,2} g_{i}\left(\sigma_{+}^{i} a+\sigma_{-}^{i} a^{\dagger}\right) \\
& +\sum_{i=1,2} \frac{\Omega_{i}}{2}\left(\sigma_{+}^{i} e^{-i \omega_{d} t}+\sigma_{-}^{i} e^{i \omega_{d} t}\right),
\end{aligned}
$$

where $\omega_{i}(i=1,2)$ are the frequencies of the qubit $Q_{i}$ and $\sigma_{z(+,-)}^{i}$ are the corresponding Pauli $Z$, raising and lowering operators. $\omega_{b}$ is the frequency of the resonator with creation and annihilation operators $a^{\dagger}$ and $a$. The drive frequency $\omega_{d}$ is detuned from the qubits and the bus as $\omega_{d}=\omega_{1(2)}+\Delta_{1(2)}=$ $\omega_{b}+\delta$. Under the conditions $g_{i} \ll \Omega_{i} \ll \Delta_{i}$ and $\delta \ll \mid \Delta_{1}-$ $\Delta_{2}$ |, Eq. (1) can be transformed into (see Appendix A)

$$
\begin{aligned}
H= & \sum_{i=1,2} \frac{\widetilde{\Delta}_{i}^{\prime}}{2} \sigma_{z}^{i}-\delta a^{\dagger} a+\sum_{i=1,2} \frac{g_{i} \Omega_{i}}{2 \Delta_{i}} \sigma_{z}^{i}\left(a^{\dagger}+a\right) \\
& +\sum_{i=1,2} \frac{g_{i}^{2}}{\widetilde{\Delta}_{i}-\delta} \sigma_{z}^{i} a^{\dagger} a,
\end{aligned}
$$

where $\widetilde{\Delta}_{i}=\Delta_{i}+\Omega_{i}^{2} /\left(2 \Delta_{i}\right), \widetilde{\Delta}_{i}^{\prime}=\widetilde{\Delta}_{i}+g_{i}^{2} /\left(\widetilde{\Delta}_{i}-\delta\right)(i=1,2)$. Ideally, since $g_{i} \ll \Omega_{i}$, the last term in Eq. (2) can be ignored. The effective Hamiltonian resembles that for the MølmerSørensen gate in an ion trap [41-43]. In the interaction picture of $H_{0}=\sum_{i=1,2} \frac{1}{2} \widetilde{\Delta}_{i}^{\prime} \sigma_{z}^{i}-\delta a^{\dagger} a$, the unitary evolution of the gate can then be derived by Magnus expansion as

$$
\begin{aligned}
& H_{\text {gate }}=\sum_{i=1,2} \frac{g_{i} \Omega_{i}}{2 \Delta_{i}} \sigma_{z}^{i}\left(a^{\dagger} e^{-i \delta t}+a e^{i \delta t}\right), \\
& U_{\text {gate }}=e^{-i H_{\text {gate }} t}=e^{\left[\alpha(t) a^{\dagger}+\alpha^{*}(t) a\right]} e^{i \beta(t)\left(\frac{g_{1} \Omega_{1}}{2 \Delta_{1}} \sigma_{z}^{1}+\frac{g_{2} \Omega_{2}}{2 \Delta_{2}} \sigma_{z}^{2}\right)^{2}},
\end{aligned}
$$

where $\alpha(t)=\sum_{i=1,2} \frac{1}{\delta} \frac{g_{i} \Omega_{i}}{2 \Delta_{i}}\left(e^{-i \delta t}-1\right) \sigma_{z}^{i}, \quad \beta(t)=\frac{1}{\delta^{2}}(-\delta t+$ $\sin \delta t)$. By setting a gate time $t_{\text {gate }}=2 n \pi / \delta$ for an integer $n$ with constant microwave amplitude satisfying 
(a)

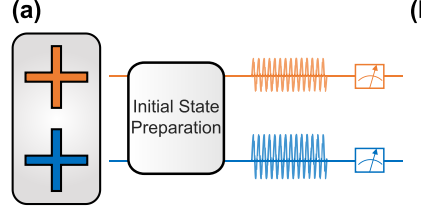

(c)

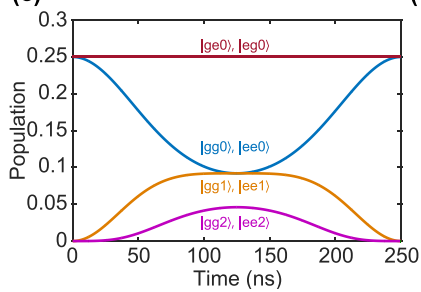

(d)

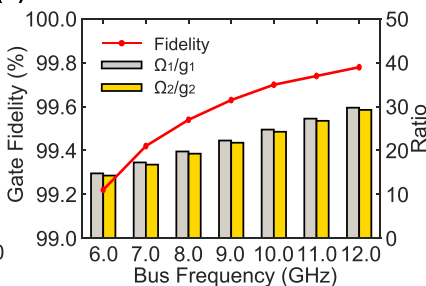

FIG. 2. (a) Schematic pulse sequence for the ideal two-qubit geometric phase gate. (b) The qubit-state-dependent phase-space trajectories of the resonator mode. (c) The ideal time evolution of the system based on the Hamiltonian in Eq. (2) without the last term starting from the initial state $(|g\rangle+|e\rangle)(|g\rangle+|e\rangle)|0\rangle / 2$. Here, $|g\rangle$ $(|e\rangle)$ and $|n\rangle(n=0,1,2, \ldots)$ represent the single-qubit ground (excited) state and cavity photon state in the bare basis. The population undergoes cyclic evolution, and each basis state acquires a different geometric phase. (d) Gate fidelity under the full Hamiltonian equation (1) vs the quality of the approximation conditions $\Omega_{i} / g_{i}$ $(i=1,2)$. High gate fidelity is achieved when $\Omega_{i} \gg g_{i}$ is satisfied.

$\left(n \pi g_{1} g_{1} \Omega_{1} \Omega_{2}\right) /\left(\delta^{2} \Delta_{1} \Delta_{2}\right)=\pi / 4$, we get a two-qubit geometric phase gate $\mathrm{GP}_{2} \equiv \exp \left[-i(\pi / 4) \sigma_{z}^{1} \sigma_{z}^{2}\right]$, which is equivalent to the controlled-NOT (CNOT) gate up to singlequbit rotations.

This ideal single-segment gate scheme is sketched in Fig. 2(a). In order to show the whole gate process more clearly, we further study the evolution of the photon in the bus. According to Eq. (3), the bus is driven classically and evolves from the ground state to the coherent state:

$$
|\psi(t)\rangle=e^{i \beta(t)\left(\frac{g_{1} \Omega_{1}}{2 \Delta_{1}} \sigma_{z}^{1}+\frac{g_{2} \Omega_{2}}{2 \Delta_{2}} \sigma_{z}^{2}\right)^{2}}|\alpha(t)\rangle .
$$

Under the constant microwave drive, the cavity mode experiences a qubit-state-dependent force in the phase space such that its trajectory has a radius $\frac{1}{\delta}\left(\frac{g_{1} \Omega_{1}}{2 \Delta_{1}} \sigma_{z}^{1}+\frac{g_{2} \Omega_{2}}{2 \Delta_{2}} \sigma_{z}^{2}\right)$ and an angular velocity $\delta$; meanwhile, it accumulates an AharonovAnandan geometric phase during this cyclic evolution, which is proportional to the enclosed area [40]. The corresponding phase-space trajectories are shown in Fig. 2(b) (here, for simplicity, we further choose $g_{1} \Omega_{1} / \Delta_{1}=g_{2} \Omega_{2} / \Delta_{2}$ such that the forces for the $|g g\rangle$ and the $|e e\rangle$ states are opposite while those for the $|g e\rangle$ and $|e g\rangle$ states vanish). Since $\sigma_{z}^{i}$ is conserved for the Hamiltonian equation (2), the population in each qubit basis state remains unchanged while the bus state undergoes cyclic evolution as shown in Fig. 2(c), which creates the desired geometric phases.

\section{B. Single-segment scheme with counter-rotating term}

When the qubit frequency is much lower than the bus frequency such as in fluxonium qubits [56,57], the rotating-wave approximation cannot be applied. Under this circumstance, the full Hamiltonian is then described as

$$
\begin{aligned}
H= & \sum_{i=1,2}-\frac{\omega_{i}}{2} \sigma_{z}^{i}+\omega_{b} a^{\dagger} a+\sum_{i=1,2} g_{i} \sigma_{x}^{i}\left(a+a^{\dagger}\right) \\
& +\sum_{i=1,2} \Omega_{i} \cos \left(\omega_{d} t\right) \sigma_{x}^{i} .
\end{aligned}
$$

Entering the interaction picture of $\omega_{d} a^{\dagger} a$ gives the Hamiltonian $H=\sum_{i=1,2}-\frac{\omega_{i}}{2} \sigma_{z}^{i}-\delta a^{\dagger} a+\sum_{i=1,2} g_{i} \sigma_{x}^{i}\left(a e^{-i \omega_{d} t}+\right.$ $\left.a^{\dagger} e^{i \omega_{d} t}\right)+\sum_{i=1,2} \frac{\Omega_{i}}{2}\left(e^{-i \omega_{d} t}+e^{i \omega_{d} t}\right) \sigma_{x}^{i}$. Since $\omega_{d}$ is the highest energy scale, we can use the formalism of Ref. [58] to obtain an effective Hamiltonian for this fast-oscillating system. Specifically, we get $H_{0}=-\sum_{i=1,2} \frac{\omega_{i}}{2} \sigma_{z}-\delta a^{\dagger} a$ and

$$
\begin{aligned}
V^{(+1)} & =\sum_{i=1,2} g_{i} \sigma_{x}^{i} a^{\dagger}+\sum_{i=1,2} \frac{\Omega_{i}}{2} \sigma_{x}^{i}, \\
V^{(-1)} & =\sum_{i=1,2} g_{i} \sigma_{x}^{i} a+\sum_{i=1,2} \frac{\Omega_{i}}{2} \sigma_{x}^{i} .
\end{aligned}
$$

The effective Hamiltonian is then expressed as

$$
H=H_{0}+\frac{1}{\omega_{d}}\left[V^{(+1)}, V^{(-1)}\right]=H_{0}-\frac{1}{\omega_{d}}\left(\sum_{i=1,2} g_{i} \sigma_{x}^{i}\right)^{2} \text {. }
$$

It is obvious that the qubit-state-dependent displacement terms no longer exist due to the commutation of the $\sigma_{x}$ terms, in other words, the cancellation between the rotating-wave and the counter-rotating-wave terms. However, there is a simple scheme to regenerate the qubit-state-dependent displacement terms. Let us consider the situation where the qubit is capacitively driven while the qubit-bus coupling is inductive, which can be realized in experiment, for example, for fluxonium qubits. Then we obtain

$$
\begin{aligned}
V^{(+1)} & =\sum_{i=1,2} g_{i} \sigma_{x}^{i} a^{\dagger}+\sum_{i=1,2} \frac{\Omega_{i}}{2} \sigma_{y}^{i}, \\
V^{(-1)} & =\sum_{i=1,2} g_{i} \sigma_{x}^{i} a+\sum_{i=1,2} \frac{\Omega_{i}}{2} \sigma_{y}^{i} .
\end{aligned}
$$

Following the derivation of Eq. (7), this time we get an effective Hamiltonian

$$
H=H_{0}+\sum_{i=1,2} \frac{g_{i} \Omega_{i}}{\omega_{d}} i \sigma_{z}^{i}\left(a^{\dagger}-a\right),
$$

where the high-order term $-\frac{1}{\omega_{d}}\left(\sum_{i=1,2} g_{i} \sigma_{x}^{i}\right)^{2}$ has been neglected considering the approximation condition $g_{i} \ll$ $\Omega_{i}(i=1,2)$. It is clear that this effective Hamiltonian is intrinsically the same as Eq. (2), with the small difference that the coefficient of the qubit-state-dependent displacement is twice as large with a $\frac{\pi}{2}$ phase. Therefore our gate scheme still applies for this case. Note that here we still consider the ideal two-level model, only with the counter-rotating term included. For realistic qubits such as fluxonium, the higher energy levels may not be neglected, and in this situation, the multisegment scheme described in Sec. III can be applied.

With this modification, now we study the quality of the approximations for this single-segment scheme numerically [59] in Fig. 2(d), where we fix $\omega_{1}=2 \pi \times 0.1 \mathrm{GHz}, \omega_{2}=2 \pi \times$ $0.3 \mathrm{GHz}, g_{1}=g_{2}=2 \pi \times 20 \mathrm{MHz}$, and $\delta=2 \pi \times 4 \mathrm{MHz}$ as 


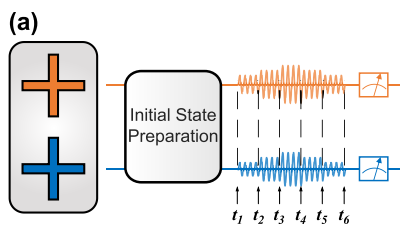

(c) Bus Frequency $(\mathrm{GHz})$

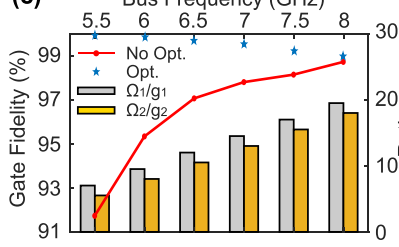

(e)
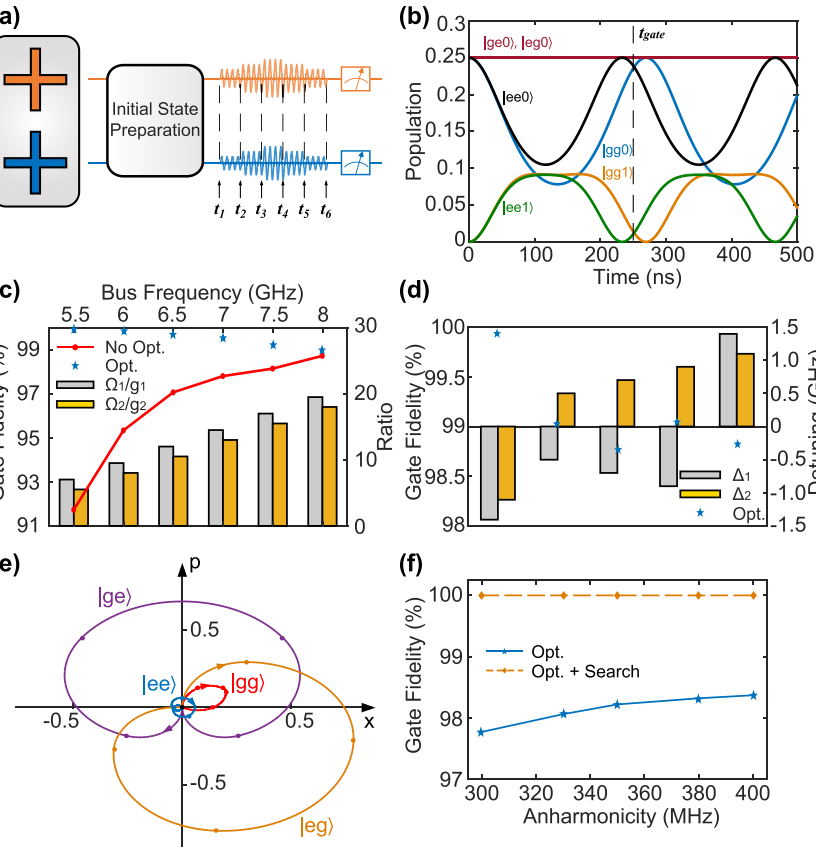

(d)

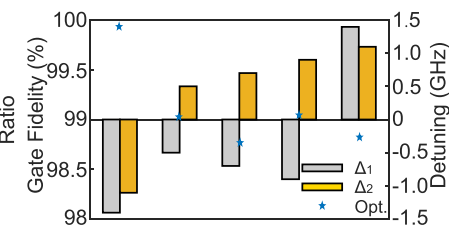

(f)

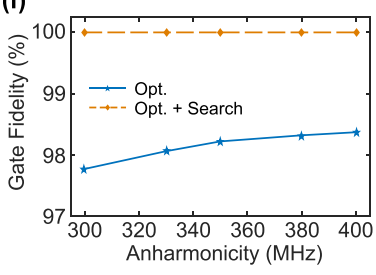

FIG. 3. (a) Schematic pulse sequence for a two-qubit geometric phase gate using $k=5$ pulse segments. (b) The time evolution of the system based on the Hamiltonian in Eq. (2) using the single-segment gate scheme in the transmon parameter regime. The system quantum state is represented in the bare basis indicated in the figure. A large deviation from the ideal case is observed due to the breakdown of the approximations. (c) Simulated gate fidelity based on the Hamiltonian in Eq. (1) for the single-segment (red dots) and multisegment ( $k=4$, blue stars) schemes at various bus frequencies and ratios of $\Omega_{i} / g_{i}$ (where $\Omega_{i}$ represents the Rabi frequency in the single-segment scheme). (d) The gate fidelity of the multisegment $(k=4)$ scheme at various bus frequencies and qubit frequencies [The first group of bars (left side): Both the qubit frequencies are lower than the bus frequencies. The fifth group of bars (right side): Both qubit frequencies are higher than the bus frequencies. The second to fourth groups of bars: The frequency of $Q_{1}\left(Q_{2}\right)$ is lower (higher) than the bus frequency]. (e) The qubit-state-dependent phase-space trajectories for the bus state using the multisegment $(k=5)$ scheme with the higher qubit levels considered. Nodes on each path separate different segments. (f) Gate fidelity vs qubit anharmonicity using the multisegment $(k=5)$ scheme with the higher qubit levels considered. The simulation is executed based on the Hamiltonian in Eq. (13). With the further "parameter-search" method to compensate the approximation errors, gate fidelity can always be optimized to above $99.99 \%$. Opt., optimization.

typical parameters for fluxonium qubits. For $\omega_{b} / 2 \pi$ ranging from 6 to $12 \mathrm{GHz}$, we design $\Omega_{1}$ and $\Omega_{2}$ accordingly using the above equations with $n=1$ and compute the gate fidelity. As the ratio $\Omega_{i} / g_{i}$ increases, we see that the gate fidelity is systematically improved.

\section{Multisegment scheme}

For typical transmon qubit [60] parameters, the above approximation conditions are not well satisfied. Hence we develop a multisegment scheme as sketched in Fig. 3(a), where we divide the total gate time $t_{\text {gate }}$ into $k$ equal segments, with piecewise constant microwave amplitudes on each segment. For example, suppose we have $\omega_{1}=2 \pi \times 4.1 \mathrm{GHz}$, $\omega_{2}=2 \pi \times 4.4 \mathrm{GHz}, \omega_{b}=2 \pi \times 5.5 \mathrm{GHz}, g_{1}=g_{2}=2 \pi \times$ $20 \mathrm{MHz}$, and $\delta=2 \pi \times 4 \mathrm{MHz}$. The designed $\Omega_{1}$ and $\Omega_{2}$ in the single-segment scheme above will give a ratio $\Omega_{i} / g_{i}$ of less than 7 and thus causes considerable gate infidelity. As we can see in Fig. 3(b), the resonator does not return to the initial state under Eq. (2) using the designed gate parameters. Note that, if we still have $g_{i}, \Omega_{i} \ll \Delta_{i}$, Eq. (2) will be valid and the dominant error in the single-segment scheme comes from the last term in Eq. (2), which can be recognized as a qubit-state-dependent frequency shift of the resonator. This can be understood as we arrange the effective Hamiltonian in Eq. (2) as

$$
H=\sum_{i=1,2} \frac{1}{2} \widetilde{\Delta}_{i}^{\prime} \sigma_{z}^{i}-\delta_{q d}^{\prime} a^{\dagger} a+\sum_{i=1,2} \frac{g_{i} \Omega_{i}}{2 \Delta_{i}} \sigma_{z}^{i}\left(a^{\dagger}+a\right),
$$

where $\delta_{q d}^{\prime}=\delta-\sum_{i=1,2} \frac{g_{i}^{2}}{\Delta_{i}-\delta} \sigma_{z}^{i}$ is the qubit-state-dependent frequency detuning of the bus. Since the qubit $|g\rangle$ and $|e\rangle$ states are conserved by this Hamiltonian and the reduced Hamiltonian in the photon subspace is quadratic in $a$ and $a^{\dagger}$, the unitary evolution as well as the obtained geometric phases can still be solved analytically. According to Eq. (10), the evolution of the photon state $|\alpha(t)\rangle$ in the Schrödinger's picture has the form

$$
\alpha(t)=\alpha(0) e^{-i \delta_{q d}^{\prime} t}+\left(e^{-i \delta_{q d}^{\prime} t}-1\right) D_{q d},
$$

where $D_{q d}=\frac{1}{\delta_{q d}^{\prime}} \sum_{i=1,2} \frac{g_{i}}{2 \Delta_{i}} \sigma_{z}^{i} \Omega_{i}$ describes the qubit-statedependent displacement. Note that in addition to the displaced state $|\alpha(t)\rangle$, there is a geometric phase that is proportional to the area enclosed by the phase-space trajectory. To realize an effective two-qubit gate operation, the photon system at the gate time should be decoupled from the qubit system. Hence, by dividing the gate sequence into $k$ segments, we obtain enough degrees of freedom to move all the qubit-statedependent phase-space trajectories back to the origin again, namely, to suppress the residual qubit-bus entanglement and to improve the gate fidelity.

By replacing $\alpha(0)$ in Eq. (11) with the state at the end of the previous segment, the final state at the gate time can be acquired as $\alpha\left(t_{\text {gate }}\right)=e^{i \theta_{i j}\left(t_{\text {gate }}\right)} \alpha(0)+C_{i j}$; here $i, j \in\{0,1\}$ denote the state of each qubit, and $\theta_{i j}$ is the aforementioned qubit-state-dependent detuning. $C_{i j}$ is the final displacement of the bus state, which is determined by the segment number, the duration time, and the amplitude of the segments. To suppress the residual qubit-bus entanglement, it is necessary to make the qubit-state-dependent photon states return to the same point in the phase space, which implies

$$
C_{00}=C_{01}=C_{10}=C_{11} \text {. }
$$

This requirement gives three complex linear equations, and thus it gives six real ones. If we use four segments $(k=4)$ for the pulses on each qubit, we get eight degrees of freedom, which are enough for these six linear constraints together with one more requirement on the two-qubit geometric phase. In Fig. 3(c) we present the optimization results using $k=4$ segments and compare the gate fidelity with that from the single-segment scheme. Note that, while the fidelity of the 
single-segment scheme is improving with the increasing of the ratio $\Omega_{i} / g_{i}$ due to the requirement $\Omega_{i} \gg g_{i}$, for the multisegment scheme here the fidelity is decreasing because now the dominant error comes from the condition $\Omega_{i}, g_{i} \ll \Delta_{i}$. Nevertheless, we see that the gate fidelity is significantly enhanced under the the multisegment scheme and can be above $99 \%$ for the available transmon parameters. In addition, in Fig. 3(d) we explore different settings for the qubit frequencies compared with the bus and find that high gate fidelity can be achieved using the multisegment scheme for various frequency setups.

\section{TWO-QUBIT GEOMETRIC PHASE GATE IN A MULTILEVEL MODEL}

Moreover, transmon qubits typically have small anharmonicity compared with the microwave detuning used above, so the existence of higher energy levels should also be considered. The system Hamiltonian now can be described as

$$
\begin{aligned}
H= & \sum_{i=1,2}\left(\omega_{i} a_{i}^{\dagger} a_{i}+\frac{\alpha_{i}}{2} a_{i}^{\dagger} a_{i}^{\dagger} a_{i} a_{i}\right)+\omega_{b} a^{\dagger} a \\
& +\sum_{i=1,2} g_{i}\left(a_{i}^{\dagger} a+a_{i} a^{\dagger}\right)+\sum_{i=1,2} \frac{\Omega_{i}}{2}\left(a_{i}^{\dagger} e^{i \omega_{d} t}+a_{i} e^{-i \omega_{d} t}\right),
\end{aligned}
$$

where $a_{i}^{\dagger}, a_{i}(i=1,2)$ are the corresponding creation and annihilation operators for the two qubits and $\alpha_{i}(i=1,2)$ are the anharmonicities of the qubit. Note that in Eq. (1) we have $\sigma_{+}=|e\rangle\langle g|$ and $\sigma_{-}=|g\rangle\langle e|$, and through the off-resonant coupling to the resonator, these terms become the qubit-statedependent force and frequency shift $(|g\rangle\langle g|-| e\rangle\langle e|)\left(a^{\dagger}+a\right)$, $(|g\rangle\langle g|-| e\rangle\langle e|) a^{\dagger} a$. Now following a similar derivation and truncated to the third energy level (detailed derivations can be found in Appendix B), we get the Hamiltonian

$$
\begin{aligned}
H= & -\delta a^{\dagger} a+\sum_{i=1,2}\left[\left(-\widetilde{\Delta}_{1}^{i}-\frac{g_{i}^{2}}{\widetilde{\Delta}_{1}^{i}-\delta}\right) \Pi_{1}^{i}\right. \\
& +\left(-\widetilde{\Delta}_{1}^{i}-\widetilde{\Delta}_{2}^{i}-\frac{2 g_{i}^{2}}{\widetilde{\Delta}_{2}^{i}-\delta}\right) \Pi_{2}^{i} \\
& +\frac{g_{i} \Omega_{i}}{2 \Delta_{i}} \sigma_{1 z}^{i}\left(a+a^{\dagger}\right)+\frac{\sqrt{2} \lambda_{i} g_{i} \Omega_{i}}{2\left(\Delta_{i}-\alpha_{i}\right)} \sigma_{2 z}^{i}\left(a+a^{\dagger}\right) \\
& \left.+\frac{g_{i}^{2}}{\widetilde{\Delta}_{1}^{i}-\delta} \sigma_{1 z}^{i} a^{\dagger} a+\frac{2 g_{i}^{2}}{\widetilde{\Delta}_{2}^{i}-\delta} \sigma_{2 z}^{i} a^{\dagger} a\right],
\end{aligned}
$$

where $\Pi_{1}^{i}=|e\rangle_{i}\left\langle e\left|, \Pi_{2}^{i}=\right| f\right\rangle_{i}\langle f|, \sigma_{1 z}^{i}=\Pi_{0}^{i}-\Pi_{1}^{i}, \sigma_{2 z}^{i}=$ $\Pi_{1}^{i}-\Pi_{2}^{i} . \widetilde{\Delta}_{1}^{i}=\Delta_{i}+\frac{\Omega_{i}^{2}}{2 \Delta_{i}}-\frac{\lambda_{i}^{2} \Omega_{i}^{2}}{4\left(\Delta_{i}-\alpha_{i}\right)}, \widetilde{\Delta}_{2}^{i}=\Delta_{i}-\alpha_{i}-\frac{\Omega_{i}^{2}}{4 \Delta_{i}}+$ $\frac{\lambda_{i}^{2} \Omega_{i}^{2}}{2\left(\Delta_{i}-\alpha_{i}\right)}(i=1,2)$ are the modified energy gaps, correspondingly. Note that the exact value of $\lambda_{i}(i=1,2)$ depends on the circuit properties and the driving format of the qubits [61]. For example, if both qubits are directly driven by the microwave pulse, $\lambda_{i}=\sqrt{2}$. Compared with the effective Hamiltonian in Eq. (10), the corresponding rotation and displacement are now modified as $\delta_{q d}^{\prime}=\delta-\sum_{i=1,2}\left(\frac{g_{i}^{2}}{\Delta_{i}-\delta} \sigma_{1 z}^{i}+\frac{2 g_{i}^{2}}{\Delta_{i}-\alpha_{i}-\delta} \sigma_{2 z}^{i}\right), D_{q d}=$ $\frac{1}{\delta_{q d}^{\prime}} \sum_{i=1,2}\left(\frac{g_{i}}{2 \Delta_{i}} \sigma_{1 z}^{i}+\frac{\sqrt{2} \lambda_{i} g_{i}}{2\left(\Delta_{i}+\alpha_{i}\right)} \sigma_{2 z}^{i}\right) \Omega_{i}$. It turns out that the coupling between $|e\rangle$ and $|f\rangle$ gives us an additional qubit-state-dependent force and frequency shift $|e\rangle\langle e|\left(a^{\dagger}+a\right)$
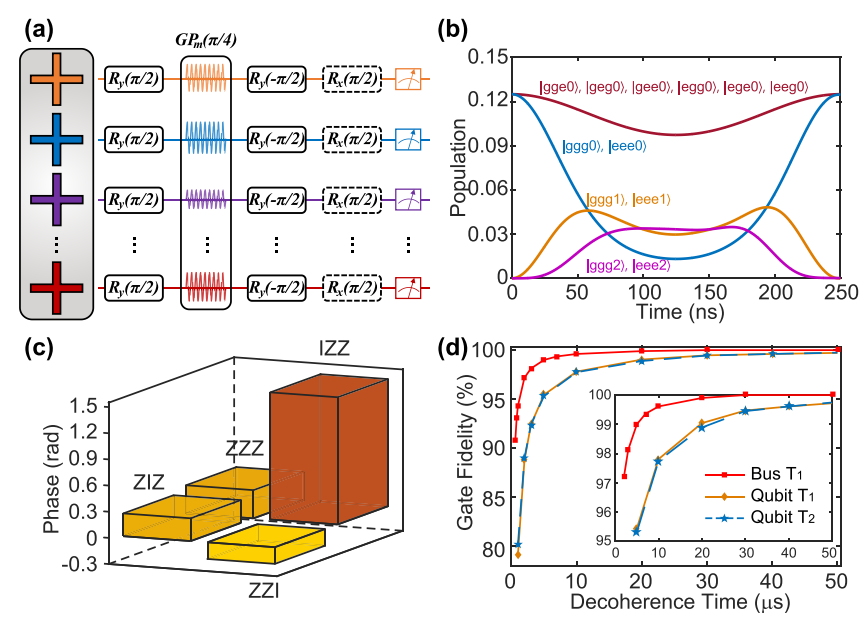

FIG. 4. (a) Schematic pulse sequence for an $m$-qubit geometric phase gate $\mathrm{GP}_{m}$ and the generation of an $m$-qubit GHZ state in a single step. The single-qubit rotation $R_{x}(\pi / 2)$ is added for odd $m$. (b) The time evolution of $\mathrm{GP}_{3}$ under the ideal two-level approximation Hamiltonian in Eq. (17) for fluxonium qubits from the initial state $(|g\rangle+|e\rangle)(|g\rangle+|e\rangle)(|g\rangle+|e\rangle)|0\rangle / 2 \sqrt{2}$. (c) Accumulated three-qubit geometric phases for the multilevel transmon model. Apart from the two-qubit phases, we also obtain a three-body $Z Z Z$ term from the qubit-state-dependent force and the frequency shift, which can be useful in quantum simulation of a many-body interaction Hamiltonian. The global phase and the single-qubit terms are not presented here. (d) Gate fidelity vs decoherence time of the system based on the Lindblad master equation [red squares, $T_{1}$ of the bus where the Lindblad operator is chosen as $a$; orange diamonds, $T_{1}$ of the qubit where the Lindblad operator is chosen as $a_{i}(i=1$ or 2$)$; blue stars, $T_{2}$ of the qubit where the Lindblad operator is chosen as $a_{i}^{\dagger} a_{i}(i=1$ or 2$\left.)\right]$. Here, we assume a total gate time $t_{\text {gate }}=500 \mathrm{~ns}$. The inset shows an enlarged region of the figure.

and $|e\rangle\langle e| a^{\dagger} a$ (the $|f\rangle\langle f|$ terms are irrelevant because the $|f\rangle$ level is never populated in our scheme). Apparently, the multisegment scheme could still work in this situation, and technically this additional displacement has not introduced any new challenge to finding a solution of the linear equations of Eq. (12). Then, in analogy to Eq. (3), $U_{\text {gate }}$ can be calculated in the interaction picture as

$$
\begin{gathered}
U_{\text {gate }}=U_{k}\left(\tau_{k}\right) \cdots U_{j}\left(\tau_{j}\right) \cdots U_{2}\left(\tau_{2}\right) U_{1}\left(\tau_{1}\right), \\
U_{j}(t)=e^{\mathcal{F}\left(\Omega_{j}, t\right)+i \beta(t)\left(\eta_{1}^{1} \sigma_{1 z}^{1}+\eta_{2}^{1} \sigma_{2 z}^{1}+\eta_{1}^{2} \sigma_{1 z}^{2}+\eta_{2}^{2} \sigma_{2 z}^{2}\right)^{2} t},
\end{gathered}
$$

where $\beta(t)=\frac{1}{\delta_{q d}^{\prime 2}}\left(-\delta_{q d}^{\prime} t+\sin \delta_{q d}^{\prime} t\right), \eta_{1}^{i}=\frac{g_{i} \Omega_{i}}{2 \Delta_{i}}, \eta_{2}^{i}=\frac{\sqrt{2} g_{i} \Omega_{i} \lambda_{i}}{2\left(\Delta_{i}+\alpha_{i}\right)}$ $(i=1,2) . \mathcal{F}\left(\Omega_{j}, t\right)$ represents the displacement operation of a photon in the bus. Here, the microwave pulse is supposed to be split into $k$ segments, and the duration time of each pulse is $\tau_{j}(j=1,2, \ldots, k)$. In fact, the $\sigma_{1 z}^{1} \sigma_{1 z}^{2}$ term is the target coupling term of the geometric phase gate, while other coupling terms in Eq. (15) may generate single-qubit rotations that can be easily compensated. Applying $U_{\text {gate }}$ on the two-qubit computational space, it is clear that all terms are in the linear space generated by $\sigma_{1 z}^{1} \otimes \sigma_{1 z}^{2}, I^{1} \otimes I^{2}, \sigma_{1 z}^{1} \otimes I^{2}$, and $I^{2} \otimes \sigma_{1 z}^{2}$. Hence one can always extract a pure $\sigma_{1 z}^{1} \sigma_{1 z}^{2}$ coupling term after correcting the single-qubit phases and global phase. Also note that when there is no anharmonicity, these added terms 
will make the force and the frequency shift independent of the qubit state, which prevents us from obtaining a nontrivial two-qubit gate. Therefore here it is desirable to have large qubit anharmonicity and to set the microwave detuning to be comparable.

We verify the corresponding results via simulation and again get high-fidelity two-qubit gates by moving all the qubit-state-dependent trajectories back to the origin by using multiple segments as sketched in Fig. 3(e). In Fig. 3(f) we choose $\omega_{1}=2 \pi \times 5.104 \mathrm{GHz}, \omega_{2}=2 \pi \times 5.204 \mathrm{GHz}, \omega_{b}=$ $2 \pi \times 5.5 \mathrm{GHz}, g_{1}=g_{2}=2 \pi \times 20 \mathrm{MHz}, \delta=2 \pi \times 4 \mathrm{MHz}$, and vary the qubit anharmonicity $\alpha_{1}=\alpha_{2}$ from $-2 \pi \times$ $300 \mathrm{MHz}$ to $-2 \pi \times 400 \mathrm{MHz}$. As we can see, the gate fidelity (blue stars) for the multisegment scheme $(k=5)$ improves with larger $\left|\alpha_{i}\right|$. Again the dominant error comes from the violation of the requirement $\Omega_{i}, g_{i} \ll \Delta_{i}$. Hence we implement a "parameter-search" method to further optimize the parameters. With fixed qubit and cavity frequencies, we regard the gate fidelity $F=f\left(\Omega_{i j}, \omega_{d}, t_{j}\right)$ computed from Eq. (13) as a function of the microwave amplitude $\Omega_{i j}(i \in$ $\{1,2\}, j \in\{1,2, \ldots, k\})$, drive frequency $\omega_{d}$, and time $t_{j}$ $(j \in\{1,2, \ldots, k\})$. Starting from the multisegment solution we find above, the gate fidelity can be iteratively improved using the standard numerical optimization algorithms [62]. As shown by the orange diamonds, the gate fidelity can be above $99.99 \%$ for each anharmonicity (actually, in the examples considered here they reach $100 \%$ within the numerical precision). Detailed parameters can be found in Appendix C.

\section{MULTIQUBIT GEOMETRIC PHASE GATE AND APPLICATIONS}

\section{A. Single-segment scheme}

Our geometric phase gate scheme can be easily generalized to multiple qubits coupled to a single microwave bus, as shown in Fig. 1(a). The system Hamiltonian based on a qubit model can be described as

$$
\begin{aligned}
H= & \omega_{b} a^{\dagger} a+\sum_{i=1,2, \ldots, m}-\frac{1}{2} \omega_{i} \sigma_{z}^{i}+g_{i}\left(\sigma_{+}^{i} a+\sigma_{-}^{i} a^{\dagger}\right) \\
& +\sum_{i=1,2, \ldots, m} \frac{\Omega_{i}}{2}\left(\sigma_{+}^{i} e^{i \omega_{d} t}+\sigma_{-}^{i} e^{-i \omega_{d} t}\right) .
\end{aligned}
$$

We address the $m$ qubits individually with microwave pulses at the same drive frequency $\omega_{d}$. After a similar unitary transformation, shown in Appendix A, and similar approximations, the effective Hamiltonian can be expressed as

$$
H=-\delta a^{\dagger} a+\sum_{i=1,2, \ldots, m} \frac{1}{2} \widetilde{\Delta}_{i}^{\prime} \sigma_{z}^{i}+\frac{g_{i} \Omega_{i}}{2 \Delta_{i}} \sigma_{z}^{i}\left(a^{\dagger}+a\right) .
$$

At the gate time, the $m$-qubit geometric phase gate is then acquired as

$$
\mathrm{GP}_{m} \equiv e^{-i \sum_{i, j \in\{1,2, \ldots, m\}, i<j} \frac{g_{i} \Omega_{i} g_{j} \Omega_{j}}{2 \Delta_{i} \Delta_{j}} \sigma_{z}^{i} \sigma_{z}^{j}} .
$$

We can further adjust the magnitude of $\Omega_{i}$ to design the specific two-qubit phases as we want. For example, in the case of a three-qubit gate, simultaneous independent control of all three phases $\sigma_{z}^{1} \otimes \sigma_{z}^{2}, \sigma_{z}^{2} \otimes \sigma_{z}^{3}, \sigma_{z}^{3} \otimes \sigma_{z}^{1}$ is achievable.
As for the case of more qubits, the number of two-body interaction terms exceeds the number of pulses, so we will need to split more pulse segments to get enough degrees of freedom. Figure 4(a) presents a scheme to create $m$-qubit GHZ state using the $\mathrm{GP}_{m}$ gate with all the $\theta_{i j}=\pi / 4$ together with single-qubit rotations. The ideal time evolution for three twolevel qubits under $\mathrm{GP}_{3}$ based on the Hamiltonian in Eq. (17) is shown in Fig. 4(b) with an initial state $(|g\rangle+|e\rangle)(|g\rangle+$ $|e\rangle)(|g\rangle+|e\rangle)|0\rangle / 2 \sqrt{2}$.

\section{B. Multisegment scheme}

Furthermore, for a typical transmon qubit, the qubitstate-dependent bus frequency shift terms cannot be ignored. Similar to the derivation for the two-qubit geometric phase gate in a multilevel model, the effective Hamiltonian in the interaction picture of the qubits is described as

$$
H=-\delta_{q d}^{\prime} a^{\dagger} a+D_{q d}\left(a+a^{\dagger}\right),
$$

where this time $\delta_{q d}^{\prime}$ and $D_{q d}$ are

$$
\begin{aligned}
\delta_{q d}^{\prime} & =\delta-\sum_{i=1,2, \ldots, m}\left(\frac{g_{i}^{2}}{\widetilde{\Delta}_{i}-\delta} \sigma_{1 z}^{i}+\frac{2 g_{i}^{2}}{\widetilde{\Delta}_{i}-\alpha_{i}-\delta} \sigma_{2 z}^{i}\right), \\
D_{q d} & =\frac{1}{\delta_{q d}^{\prime}} \sum_{i=1,2, \ldots, m}\left(\frac{g_{i}}{2 \Delta_{i}} \sigma_{1 z}^{i}+\frac{\sqrt{2} \lambda_{i} g_{i}}{2\left(\Delta_{i}+\alpha_{i}\right)} \sigma_{2 z}^{i}\right) \Omega_{i} .
\end{aligned}
$$

Similarly, the evolution operator in the interaction picture reveals

$$
U_{j}(t)=e^{\mathcal{F}^{\prime}\left(\Omega_{j}, t\right)+i\left(-\delta_{q d}^{\prime} t+\sin \delta_{q d}^{\prime} t\right) D_{q d}^{2} t},
$$

where $\mathcal{F}^{\prime}\left(\Omega_{j}, t\right)$ represents the displacement of the photon in the bus. Then, in analogy to Eq. (15), the $U_{\text {gate }}$ could be described as $U_{\text {gate }}=U_{k}\left(\tau_{k}\right) \cdots U_{j}\left(\tau_{j}\right) \cdots U_{2}\left(\tau_{2}\right) U_{1}\left(\tau_{1}\right)$. Note that in $U_{\text {gate }}$, the $D_{q d}^{2}$ term contains two-body interaction, and thus three-body interaction could be generated from $\delta_{q d}^{\prime} t_{j} D_{q d}^{2}$. In addition, note that the matrix form of $\sin \left(\delta_{q d}^{\prime} t_{j}\right)(j=$ $1,2, \ldots, k)$ could be projected onto any basis $\left(I_{1}, \sigma_{z}^{1}\right) \otimes$ $\left(I_{2}, \sigma_{z}^{2}\right) \otimes \cdots \otimes\left(I_{m}, \sigma_{z}^{m}\right)$, which implies interactions beyond three-body ones could also be generated. Hence the qubitstate-dependent frequency shift can also be exploited to engineer many-body interaction for quantum simulation beyond the two-body terms in the $\mathrm{GP}_{m}$ gate. In general, each qubit state in the $\{|g\rangle,|e\rangle\}^{\otimes m}$ basis can acquire a different geometric phase under the multisegment scheme, so the whole geometric phase gate can be decomposed into a global phase, $m$ single-qubit phases, $m(m-1) / 2$ two-qubit phases, ..., and an $m$-body phase. As an example, Fig. 4(c) shows the values of the two-qubit and the three-qubit phases using parameters $\omega_{1}=2 \pi \times 5.074 \mathrm{GHz}$, $\omega_{2}=2 \pi \times 5.144 \mathrm{GHz}, \omega_{3}=2 \pi \times 5.214 \mathrm{GHz}, \omega_{b}=2 \pi \times$ $5.5 \mathrm{GHz}, g_{1}=g_{2}=g_{3}=2 \pi \times 20 \mathrm{MHz}, \delta=2 \pi \times 4 \mathrm{MHz}$, and $\alpha_{1}=\alpha_{2}=\alpha_{3}=-2 \pi \times 380 \mathrm{MHz}$ with the segmented microwave amplitudes deliberately designed to enlarge the three-qubit term.

\section{DISCUSSION AND CONCLUSIONS}

To sum up, we propose a geometric phase gate scheme in a multiqubit architecture with simple implementation and 
TABLE I. Simulation parameters for the $\mathrm{GP}_{2}$. All data except for those of $t_{\text {gate }}$ are divided by $2 \pi$.

\begin{tabular}{|c|c|c|c|c|c|c|c|c|c|c|c|c|}
\hline Index & $\omega_{1}(\mathrm{GHz})$ & $\omega_{2}(\mathrm{GHz})$ & $\omega_{b}(\mathrm{GHz})$ & $g_{1}(\mathrm{MHz})$ & $g_{2}(\mathrm{MHz})$ & $\Omega_{1}(\mathrm{MHz})$ & $\Omega_{2}(\mathrm{MHz})$ & $\delta(\mathrm{MHz})$ & $\Delta_{1}(\mathrm{MHz})$ & $\Delta_{2}(\mathrm{MHz})$ & $t_{\text {gate }}(\mathrm{ns})$ & Parameter scop \\
\hline 1 & 0.1 & 0.3 & 6.0 & 20 & 20 & 295.2 & 285.2 & 4 & 5904 & 5704 & 250 & Fluxonium \\
\hline 2 & 0.1 & 0.3 & 7.0 & 20 & 20 & 345.2 & 335.2 & 4 & 6904 & 6704 & 250 & Fluxonium \\
\hline 3 & 0.1 & 0.3 & 8.0 & 20 & 20 & 395.2 & 385.2 & 4 & 7904 & 7704 & 250 & Fluxonium \\
\hline 4 & 0.1 & 0.3 & 9.0 & 20 & 20 & 445.2 & 435.2 & 4 & 8904 & 8704 & 250 & Fluxonium \\
\hline 5 & 0.1 & 0.3 & 10.0 & 20 & 20 & 495.2 & 485.2 & 4 & 9904 & 9704 & 250 & Fluxonium \\
\hline 6 & 0.1 & 0.3 & 11.0 & 20 & 20 & 545.2 & 535.2 & 4 & 10904 & 10704 & 250 & Fluxonium \\
\hline 7 & 0.1 & 0.3 & 12.0 & 20 & 20 & 595.2 & 585.2 & 4 & 11904 & 11704 & 250 & Fluxonium \\
\hline 8 & 4.1 & 4.4 & 5.5 & 20 & 20 & $140.4^{\mathrm{b}}$ & $110.4^{\mathrm{b}}$ & 4 & 1404 & 1104 & 250 & Transmon \\
\hline 9 & 4.1 & 4.4 & 6.0 & 20 & 20 & $190.4^{\mathrm{b}}$ & $160.4^{\mathrm{b}}$ & 4 & 1904 & 1604 & 250 & Transmon \\
\hline 10 & 4.1 & 4.4 & 6.5 & 20 & 20 & $240.4^{\mathrm{b}}$ & $210.4^{\mathrm{b}}$ & 4 & 2404 & 2104 & 250 & Transmon \\
\hline 11 & 4.1 & 4.4 & 7.0 & 20 & 20 & $290.4^{\mathrm{b}}$ & $260.4^{\mathrm{b}}$ & 4 & 2904 & 2604 & 250 & Transmon \\
\hline 12 & 4.1 & 4.4 & 7.5 & 20 & 20 & $340.4^{\mathrm{b}}$ & $310.4^{\mathrm{b}}$ & 4 & 3404 & 3104 & 250 & Transmon \\
\hline 13 & 4.1 & 4.4 & 8.0 & 20 & 20 & $390.4^{\mathrm{b}}$ & $360.4^{\mathrm{b}}$ & 4 & 3904 & 3604 & 250 & Transmon \\
\hline 14 & 5.004 & 6.004 & 5.5 & 20 & 20 & $\mathrm{~b}$ & b & 4 & 500 & -500 & 320 & Transmon \\
\hline 15 & 4.804 & 6.204 & 5.5 & 20 & 20 & b & b & 4 & 700 & -700 & 320 & Transmon \\
\hline 16 & 4.604 & 6.404 & 5.5 & 20 & 20 & b & b & 4 & 900 & -900 & 320 & Transmon \\
\hline 17 & 6.9 & 6.6 & 5.5 & 20 & 20 & b & b & 4 & -1396 & -1096 & 320 & Transmon \\
\hline $18-22$ & 5.104 & 5.204 & 5.5 & 20 & 20 & b & $\mathrm{b}$ & 4 & 400 & 300 & 500 & Transmon \\
\hline 23 & 5.104 & 5.204 & 5.5 & 20 & 20 & b & b & 4.077 & 400 & 300 & 501.40 & Transmon \\
\hline 24 & 5.104 & 5.204 & 5.5 & 20 & 20 & b & b & 4.711 & 400 & 300 & 500.39 & Transmon \\
\hline 25 & 5.104 & 5.204 & 5.5 & 20 & 20 & b & b & 5.138 & 400 & 300 & 500.17 & Transmon \\
\hline 26 & 5.104 & 5.204 & 5.5 & 20 & 20 & b & $\mathrm{b}$ & 4.995 & 400 & 300 & 500.31 & Transmon \\
\hline 27 & 5.104 & 5.204 & 5.5 & 20 & 20 & b & b & 5.066 & 400 & 300 & 500.17 & Transmon \\
\hline
\end{tabular}

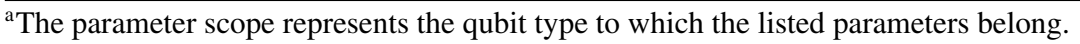

${ }^{\mathrm{b}}$ These parameters are further optimized using multiple segments in Tables II and III.

high fidelity using the available parameters for transmon and fluxonium qubits. Our scheme uses microwave-only control on fixed-frequency qubits and only the lowest qubit levels, and thus it is convenient for experiments and is not subjected to the main decoherence sources in previous schemes. If selective gates among arbitrary subgroups of qubits are desired, one can either use the multisegment scheme to set all the multiqubit geometric phases to the target value or take advantage of the recently developed tunable couplers $[63,64]$ to turn off the coupling between the other irrelevant qubits and the bus. Meanwhile, the geometric nature of this phase gate makes it robust against certain control errors and noise $[13,26,40]$. As

TABLE II. Drive amplitude for the $\mathrm{GP}_{2}$ in the multisegment scheme. All data are divided by $2 \pi$. Values are given in megahertz.

\begin{tabular}{|c|c|c|c|c|c|c|c|c|c|c|}
\hline Index & $\Omega_{11}$ & $\Omega_{12}$ & $\Omega_{13}$ & $\Omega_{14}$ & $\Omega_{15}$ & $\Omega_{21}$ & $\Omega_{22}$ & $\Omega_{23}$ & $\Omega_{24}$ & $\Omega_{25}$ \\
\hline 8 & 72.21 & 133.48 & 137.40 & 72.25 & & -70.80 & -130.74 & -126.86 & -70.23 & \\
\hline 9 & 100.45 & 186.01 & 188.03 & 100.56 & & -99.78 & -184.62 & -182.60 & -99.57 & \\
\hline 10 & 129.07 & 239.02 & 240.25 & 129.15 & & -128.67 & -238.18 & -236.95 & -128.56 & \\
\hline 11 & 157.59 & 291.81 & 292.64 & 157.65 & & -157.32 & -291.25 & -290.42 & -157.25 & \\
\hline 12 & 186.30 & 344.97 & 345.57 & 186.35 & & -186.11 & -344.57 & -343.98 & -186.07 & \\
\hline 13 & 214.98 & 398.08 & 398.52 & 215.02 & & -214.84 & -397.77 & -397.33 & -214.81 & \\
\hline 14 & -28.84 & -53.40 & -53.40 & -28.84 & & 28.84 & 53.40 & 53.40 & 28.84 & \\
\hline 15 & -40.41 & -74.81 & -74.81 & -40.41 & & 40.41 & 74.81 & 74.81 & 40.41 & \\
\hline 16 & 51.81 & 95.92 & 95.92 & 51.81 & & -51.81 & -95.92 & -95.92 & -51.81 & \\
\hline 17 & -71.66 & -132.46 & -136.42 & -71.70 & & 70.24 & 129.69 & 125.76 & 69.65 & \\
\hline 18 & 23.33 & 49.55 & 68.37 & 49.55 & 23.33 & -24.67 & -42.84 & -52.78 & -42.84 & -24.67 \\
\hline 19 & -19.75 & -48.24 & -64.17 & -48.24 & -19.75 & 24.50 & 40.15 & 53.85 & 40.15 & 24.50 \\
\hline 20 & 17.12 & 47.95 & 61.47 & 47.95 & 17.12 & -23.49 & -40.00 & -52.71 & -40.00 & -23.49 \\
\hline 21 & 14.79 & 46.87 & 59.48 & 46.87 & 14.79 & -21.57 & -40.22 & -50.01 & -40.22 & -21.57 \\
\hline 22 & 13.88 & 45.93 & 58.80 & 45.93 & 13.88 & -20.52 & -40.13 & -48.52 & -40.13 & -20.52 \\
\hline 23 & 28.23 & 48.21 & 75.37 & 46.83 & 28.44 & -25.91 & -38.48 & -58.16 & -38.46 & -23.62 \\
\hline 24 & -16.17 & -48.54 & -83.69 & -71.82 & -33.72 & 8.65 & 34.46 & 59.88 & 50.43 & 25.04 \\
\hline 25 & 18.55 & 57.55 & 85.72 & 64.70 & 23.06 & -13.46 & -43.81 & -66.05 & -49.36 & -17.57 \\
\hline 26 & 14.03 & 47.82 & 80.51 & 71.74 & 29.98 & -11.27 & -33.70 & -60.41 & -49.63 & -21.77 \\
\hline 27 & 19.28 & 53.76 & 75.24 & 60.99 & 25.30 & -17.04 & -41.47 & -61.60 & -46.73 & -19.87 \\
\hline
\end{tabular}


we can see in Fig. 4(d), the gate fidelity is quite insensitive to decoherence of both the qubit and the resonator. Furthermore, since the evolution of the qubit is restricted to the subspace of the lowest two levels, there is no limit from the relatively short coherence time of the higher qubit levels. Our multiqubit geometric phase gate scheme thus provides an attractive approach to fault-tolerant quantum computing, as well as a natural way to create many-body interactions for quantum simulation of complicated spin models.

\section{ACKNOWLEDGMENTS}

We thank Haonan Xiong, Yuwei Ma, Xuegang Li, and Minglei Cai for valuable discussions. This work is supported by National Natural Science Foundation of China under Grants No. 11874235 and No. 11674060. Y.-K.W. acknowledges in addition support from the Shuimu Tsinghua Scholar Program and International Postdoctoral Exchange Fellowship Program.

\section{APPENDIX A: FULL DERIVATION FOR THE TWO-QUBIT GEOMETRIC PHASE GATE IN A QUBIT MODEL}

In this Appendix, we develop the theory of the two-qubit geometric phase gate based on a two-level qubit model. Our system consists of two superconducting qubits $Q_{1}$ and $Q_{2}$ weakly coupling to a single-mode harmonic oscillator with coupling strength $g_{i}(i=1,2)$. We consider a system which satisfies the rotating-wave approximation (RWA). The system with the counter-rotating term has been fully derived in Sec. II. The static Hamiltonian can be represented as $(\hbar=1)$

$$
H_{s}=\sum_{i=1,2}-\frac{1}{2} \omega_{i} \sigma_{z}^{i}+\omega_{b} a^{\dagger} a+\sum_{i=1,2} g_{i}\left(\sigma_{+}^{i} a+\sigma_{-}^{i} a^{\dagger}\right),
$$

where $\omega_{i}(i=1,2)$ are the frequencies of the qubit $Q_{i}$ and $\sigma_{z(+,-)}^{i}$ are the corresponding Pauli $Z$, raising and lowering operators. $\omega_{b}$ is the frequency of the resonator with creation and annihilation operators $a^{\dagger}$ and $a$. When both qubits are individually driven via their own independent XY control line by a microwave tone at the same drive frequency $\omega_{d}$ but with different drive amplitudes $\Omega_{i}(i=1,2)$, the drive Hamiltonian under the rotating-wave approximation can be expressed as

$$
H_{d}=\sum_{i=1,2} \frac{\Omega_{i}}{2}\left(\sigma_{+}^{i} e^{-i \omega_{d} t}+\sigma_{-}^{i} e^{i \omega_{d} t}\right) .
$$

Here, the drive frequency $\omega_{d}$ satisfies the condition $\omega_{d}=$ $\omega_{1(2)}+\Delta_{1(2)}=\omega_{b}+\delta$. Thus the full system Hamiltonian is the combination of the static Hamiltonian and the drive Hamiltonian

$$
H=H_{s}+H_{d}
$$

Transforming the system Hamiltonian into the rotating frame at the drive frequency $\omega_{d}$ via the unitary transformation

$$
U_{1}=e^{i \omega_{d} t\left(\frac{\sigma_{z}^{1}}{2}+\frac{\sigma_{z}^{2}}{2}-a^{\dagger} a\right)},
$$

we get

$$
\begin{aligned}
H^{\prime}= & U^{\dagger} H U-i U^{\dagger} \dot{U} \\
T= & \sum_{i=1,2} \frac{1}{2} \Delta_{i} \sigma_{z}^{i}-\delta a^{\dagger} a+\sum_{i=1,2} g_{i}\left(\sigma_{+}^{i} a+\sigma_{-}^{i} a^{\dagger}\right) \\
& +\sum_{i=1,2} \frac{\Omega_{i}}{2}\left(\sigma_{+}^{i}+\sigma_{-}^{i}\right) .
\end{aligned}
$$

During the gate operation, the driving terms hybridize the bare qubits' energy levels. To clearly view the physical process in the basis of dressed states in the weak driving condition, $\Omega_{i} \ll$ $\Delta_{i}(i=1,2)$, the Hamiltonian can be rotated with the unitary transformation

$$
U_{2}=e^{\frac{\Omega_{1}}{2 \Delta_{1}}\left(\sigma_{+}^{1}-\sigma_{-}^{1}\right)+\frac{\Omega_{2}}{2 \Delta_{2}}\left(\sigma_{+}^{2}-\sigma_{-}^{2}\right)} .
$$

Retaining this to the second order gives the Hamiltonian

$$
\begin{aligned}
H^{\prime \prime}= & \sum_{i=1,2} \frac{1}{2} \widetilde{\Delta}_{i} \sigma_{z}^{i}-\delta a^{\dagger} a+\sum_{i=1,2} g_{i}\left(\sigma_{+}^{i} a+\sigma_{-}^{i} a^{\dagger}\right) \\
& +\sum_{i=1,2} \frac{g_{i} \Omega_{i}}{2 \Delta_{i}} \sigma_{z}^{i}\left(a^{\dagger}+a\right),
\end{aligned}
$$

where $\widetilde{\Delta}_{i}=\Delta_{i}+\frac{\Omega_{i}^{2}}{2 \Delta_{i}}(i=1,2)$. Obviously, after this transformation, the off-resonance driving for qubits is manifested as a qubit-state-dependent displacement for the bus. In our geometric phase gate regime, both of the qubits are dispersively coupled to the common bus with $g_{i} \ll \Delta_{i}, \delta \ll \Delta_{i},\left|\Delta_{1}-\Delta_{2}\right|(i=1,2)$. Therefore the Hamiltonian in the dispersive regime can be further transformed by the Schrieffer-Wolff transformation (SWT) [65]

$$
U_{3}=e^{\frac{g_{1}}{\Delta_{1}-\delta}\left(\sigma_{+}^{1} a-\sigma_{-}^{1} a^{\dagger}\right)+\frac{g_{2}}{\Delta_{2}-\delta}\left(\sigma_{+}^{2} a-\sigma_{-}^{2} a^{\dagger}\right)} .
$$

The effective Hamiltonian for the two-qubit geometric phase gate is finally acquired as

$$
\begin{aligned}
H^{\prime \prime \prime}= & \sum_{i=1,2} \frac{1}{2} \widetilde{\Delta}_{i} \sigma_{z}^{i}-\delta a^{\dagger} a+\sum_{i=1,2} \frac{g_{i} \Omega_{i}}{2 \Delta_{i}} \sigma_{z}^{i}\left(a^{\dagger}+a\right) \\
& +\sum_{i=1,2} \frac{g_{i}^{2}}{2\left(\widetilde{\Delta}_{i}-\delta\right)}\left(2 \sigma_{z}^{i} a^{\dagger} a+\sigma_{z}^{i}\right) \\
& -\frac{g_{1} g_{2}}{\widetilde{\Delta}}\left(\sigma_{+}^{1} \sigma_{-}^{2}+\sigma_{-}^{1} \sigma_{+}^{2}\right),
\end{aligned}
$$

where $\widetilde{\Delta}=2 /\left(1 / \widetilde{\Delta}_{1}+1 / \widetilde{\Delta}_{2}\right)$. In this part, we consider the situation where $g_{i} \ll \Omega_{i}(i=1,2)$, which generates the results $\frac{g_{i}^{2}}{\left(\widetilde{\Delta}_{i}-\delta\right)}, \frac{g_{1} g_{2}}{\Delta} \ll \frac{g_{i} \Omega_{i}}{2 \Delta_{i}}$. Thus the last two terms in Eq. (A9) can be omitted, and the effective Hamiltonian is obtained as

$$
H_{\text {ideal }}=\sum_{i=1,2} \frac{1}{2} \widetilde{\Delta}_{i}^{\prime} \sigma_{z}^{i}-\delta a^{\dagger} a+\sum_{i=1,2} \frac{g_{i} \Omega_{i}}{2 \Delta_{i}} \sigma_{z}^{i}\left(a^{\dagger}+a\right) \text {, }
$$

where $\widetilde{\Delta}_{i}^{\prime}=\widetilde{\Delta}_{i}+g_{i}^{2} /\left(\widetilde{\Delta}_{i}-\delta\right)(i=1,2)$. In the interaction picture of $H_{0}=\sum_{i=1,2} \frac{1}{2} \widetilde{\Delta}_{i}^{\prime} \sigma_{z}^{i}-\delta a^{\dagger} a$, the gate Hamiltonian is then represented as

$$
H_{\text {gate }}=\sum_{i=1,2} \frac{g_{i} \Omega_{i}}{2 \Delta_{i}} \sigma_{z}^{i}\left(a^{\dagger} e^{-i \delta t}+a e^{i \delta t}\right) .
$$


The unitary evolution of the gate can then be derived by Magnus expansion as

$$
U_{\text {gate }}=e^{\left[\alpha(t) a^{\dagger}+\alpha^{*}(t) a\right]} e^{i \beta(t)\left(\frac{g_{1} \Omega_{1}}{2 \Delta_{1}} \sigma_{z}^{1}+\frac{g_{2} \Omega_{2}}{2 \Delta_{2}} \sigma_{z}^{2}\right)^{2}},
$$

where $\alpha(t)=\sum_{i=1,2} \frac{1}{\delta} \frac{g_{i} \Omega_{i}}{2 \Delta_{i}}\left(e^{-i \delta t}-1\right) \sigma_{z}^{i}, \beta(t)=\frac{1}{\delta^{2}}(-\delta t+\sin \delta t)$. For the purpose of realizing a two-qubit gate, the gate should not induce entanglement between the qubits and the bus. Therefore the evolution time could be chosen to satisfy $\alpha(t)=0$, and this gives the condition $t=n T(n \in Z), T=\frac{2 \pi}{\delta}$. Under this circumstance, $\beta(t)=-\frac{2 \pi n}{\delta^{2}}(n \in Z)$, and the unitary evolution becomes

$$
\begin{aligned}
U_{\text {gate }} & =e^{-i \frac{2 \pi n}{\delta^{2}}\left(\frac{g_{1} \Omega_{1}}{2 \Delta_{1}} \sigma_{z}^{1}+\frac{g_{2} \Omega_{2}}{2 \Delta_{2}} \sigma_{z}^{2}\right)^{2}} \\
& =e^{-i \frac{2 \pi n}{\delta^{2}}\left[\left(\frac{g_{1} \Omega_{1}}{2 \Delta_{1}}\right)^{2}\left(\sigma_{z}^{1}\right)^{2}+\left(\frac{g_{2} \Omega_{2}}{2 \Delta_{2}}\right)^{2}\left(\sigma_{z}^{2}\right)^{2}+\frac{g_{1} \Omega_{1} g_{2} \Omega_{2}}{2 \Delta_{1} \Delta_{2}} \sigma_{z}^{1} \sigma_{z}^{2}\right]} .
\end{aligned}
$$

It is easy to find that $\left(\sigma_{z}^{1}\right)^{2}=I^{1},\left(\sigma_{z}^{2}\right)^{2}=I^{2}\left(I^{1}, I^{2}\right.$ denote the identity operator for each of the qubits), and thus $\left(\sigma_{z}^{1}\right)^{2},\left(\sigma_{z}^{2}\right)^{2}$ terms only create a global phase for the whole gate. The remaining $\sigma_{z}^{1} \sigma_{z}^{2}$ term generates an effective $Z Z$ interaction between the two qubits which can be used to realize the geometric phase gate as we set

$$
\delta=2 \sqrt{n} \sqrt{\frac{g_{1} \Omega_{1} g_{2} \Omega_{2}}{\Delta_{1} \Delta_{2}}} .
$$

The two-qubit geometric phase gate is then acquired as

$$
\mathrm{GP}_{2} \equiv U_{\text {gate }, t=n T}=e^{-i \frac{\pi}{4} \sigma_{z}^{1} \sigma_{z}^{2}}=\left(\begin{array}{cccc}
1 & 0 & 0 & 0 \\
0 & i & 0 & 0 \\
0 & 0 & i & 0 \\
0 & 0 & 0 & 1
\end{array}\right)
$$

In fact, with single-qubit phase rotation, we can easily find that the two-qubit geometric phase $\left(\mathrm{GP}_{2}\right)$ gate turns into a controlled- $Z(\mathrm{CZ})$ gate through $\mathrm{CZ}=\left[R_{z}\left(-\frac{\pi}{2}\right) \otimes R_{z}\left(-\frac{\pi}{2}\right)\right]$. $\mathrm{GP}_{2}$, and thus is equivalent to the controlled-NOT (CNOT) gate which is essential in universal quantum computation.

\section{APPENDIX B: FULL DERIVATION FOR THE TWO-QUBIT GEOMETRIC PHASE GATE IN A MULTILEVEL MODEL}

Through the multisegment scheme, when the last term in Eq. (2) cannot be ignored, gate operations can still be well optimized under a qubit model. However, for superconducting qubits such as the transmon, we have to consider the significant impact arising from the existence of the higher energy levels. Careful investigation finds that in our geometric phase gate framework, three-level approximation is sufficient since the initial state of the qubit is always in $|g\rangle$ and $|e\rangle$ and will not evolve to a higher excited state. Based on the transmon system, with anharmonicities of both qubits in mind, we can describe the system Hamiltonian as [60]

$$
\begin{aligned}
H= & \sum_{i=1,2}\left(\omega_{i} a_{i}^{\dagger} a_{i}+\frac{\alpha_{i}}{2} a_{i}^{\dagger} a_{i}^{\dagger} a_{i} a_{i}\right)+\omega_{b} a^{\dagger} a \\
& +\sum_{i=1,2} g_{i}\left(a_{i}^{\dagger} a+a_{i} a^{\dagger}\right)+\sum_{i=1,2} \frac{\Omega_{i}}{2}\left(a_{i}^{\dagger} e^{i \omega_{d} t}+a_{i} e^{-i \omega_{d} t}\right),
\end{aligned}
$$

where $a_{i}^{\dagger}, a_{i}(i=1,2)$ are the corresponding creation and annihilation operators for the two qubits. $\alpha_{i}(i=1,2)$ are the anharmonicities of the qubit. The system Hamiltonian in the frame rotating at the drive frequency $\omega_{d}$ becomes

$$
\begin{aligned}
H^{\prime}= & -\delta a^{\dagger} a+\sum_{i=1,2}\left[-\Delta_{i} \Pi_{1}^{i}-\left(2 \Delta_{i}-\alpha_{i}\right) \Pi_{2}^{i}\right] \\
& +\sum_{i=1,2} g_{i}\left(\sigma_{1+}^{i} a+\sigma_{1-}^{i} a^{\dagger}\right)+\sqrt{2} g_{i}\left(\sigma_{2+}^{i} a+\sigma_{2-}^{i} a^{\dagger}\right) \\
& +\sum_{i=1,2} \frac{\Omega_{i}}{2}\left(\sigma_{1+}^{i}+\sigma_{1-}^{i}\right)+\frac{\lambda_{i} \Omega_{i}}{2}\left(\sigma_{2+}^{i}+\sigma_{2-}^{i}\right),
\end{aligned}
$$

where $\quad \Pi_{1}^{i}=|e\rangle_{i}\left\langle e\left|, \Pi_{2}^{i}=\right| f\right\rangle_{i}\left\langle f\left|, \quad \sigma_{1+}^{i}=\right| e\right\rangle_{i}\langle g|, \quad \sigma_{1-}^{i}=$ $|g\rangle_{i}\left\langle e\left|, \quad \sigma_{2+}^{i}=\right| f\right\rangle_{i}\left\langle e\left|, \sigma_{2-}^{i}=\right| e\right\rangle_{i}\langle f|(i=1,2) . \lambda_{i}(i=1,2)$ depends on the circuit properties and the driving format of the qubits [61]. Similarly, given the approximate condition $\Omega_{1(2)} \ll \Delta_{1(2)}$, the Hamiltonian can be rotated via the unitary transformation $U_{2}=e^{\sum_{i=1,2} \frac{\Omega_{i}}{2 \Delta_{i}}\left(\sigma_{1+}^{i}-\sigma_{1-}^{i}\right)+\frac{\lambda_{i} \Omega_{i}}{2\left(2 \Delta_{i}-\alpha_{i}\right)}\left(\sigma_{2+}^{i}-\sigma_{2-}^{i}\right)}$ as

$$
\begin{aligned}
H^{\prime \prime}=-\delta a^{\dagger} a+\sum_{i=1,2} & \left\{\frac{\Omega_{i}^{2}}{4 \Delta_{i}} \Pi_{0}^{i}+\left(-\widetilde{\Delta}_{1}^{i}+\frac{\Omega_{i}^{2}}{4 \Delta_{i}}\right) \Pi_{i}^{1}+\left(-\widetilde{\Delta}_{1}^{i}+\frac{\Omega_{i}^{2}}{4 \Delta_{i}}-\widetilde{\Delta}_{2}^{i}\right) \Pi_{2}^{i}\right. \\
& +\frac{g_{i} \Omega_{i}}{2 \Delta_{i}} \sigma_{1 z}^{i}\left(a+a^{\dagger}\right)+\frac{\sqrt{2} \lambda_{i} g_{i} \Omega_{i}}{2\left(\Delta_{i}-\alpha_{i}\right)} \sigma_{2 z}^{i}\left(a+a^{\dagger}\right)+g_{i}\left(\sigma_{1+}^{i} a+\sigma_{1-}^{i} a^{\dagger}\right)+\sqrt{2} g_{i}\left(\sigma_{2+}^{i} a+\sigma_{2-}^{i} a^{\dagger}\right) \\
& \left.\left.+\left[\frac{\lambda_{i} g_{i} \Omega_{i}}{2\left(-\Delta_{i}+\alpha_{i}\right)}+\frac{\sqrt{2} g_{i} \Omega_{i}}{2 \Delta_{i}}\right]\left(|2\rangle_{i}\langle 0|a+| 0\rangle_{i}\langle 2| a^{\dagger}\right)+\frac{\lambda_{i} \alpha_{i} \Omega_{i}^{2}}{8 \Delta_{i}\left(\alpha_{i}-\Delta_{i}\right)}|0\rangle_{i}\langle 2|+| 2\rangle_{i}\langle 0|\right)\right\},
\end{aligned}
$$

where $\Pi_{0}^{i}=|g\rangle_{i}\langle g|, \quad \sigma_{1 z}^{i}=\Pi_{0}^{i}-\Pi_{1}^{i}, \sigma_{2 z}^{i}=\Pi_{1}^{i}-\Pi_{2}^{i}, \quad \widetilde{\Delta}_{1}^{i}=\Delta_{i}+\frac{\Omega_{i}^{2}}{2 \Delta_{i}}-\frac{\lambda_{i}^{2} \Omega_{i}^{2}}{4\left(\Delta_{i}-\alpha_{i}\right)}, \widetilde{\Delta}_{2}^{i}=\Delta_{i}-\alpha_{i}-\frac{\Omega_{i}^{2}}{4 \Delta_{i}}+\frac{\lambda_{i}^{2} \Omega_{i}^{2}}{2\left(\Delta_{i}-\alpha_{i}\right)}(i=1,2)$. In the dispersive regime $g_{i} \ll \widetilde{\Delta}_{1}^{i}, \widetilde{\Delta}_{2}^{i}(i=1,2)$, the Hamiltonian can be further moved into the frame with unitary transformation $U_{3}=e^{\sum_{i=1,2} \frac{g_{i}}{\widetilde{\Delta}_{1}^{i}-\delta}\left(\sigma_{1+}^{i} a-\sigma_{1-}^{i} a^{\dagger}\right)+\frac{\sqrt{2} g_{1}}{\widetilde{\Delta}_{2}^{i}-\delta}\left(\sigma_{2+}^{i} a-\sigma_{2-}^{i} a^{\dagger}\right)}$, which gives the effective Hamiltonian $H^{\prime \prime \prime}=H_{\mathrm{eff}}+H_{\mathrm{err}}$,

$$
H_{\mathrm{eff}}=-\delta a^{\dagger} a+\sum_{i=1,2}\left[\frac{\Omega_{i}^{2}}{4 \Delta_{i}} \Pi_{0}^{i}+\left(-\widetilde{\Delta}_{1}^{i}+\frac{\Omega_{i}^{2}}{4 \Delta_{i}}-\frac{g_{i}^{2}}{\widetilde{\Delta}_{1}^{i}-\delta}\right) \Pi_{1}^{i}+\left(-\widetilde{\Delta}_{1}^{i}+\frac{\Omega_{i}^{2}}{4 \Delta_{i}}-\widetilde{\Delta}_{2}^{i}-\frac{2 g_{i}^{2}}{\widetilde{\Delta}_{2}^{i}-\delta}\right) \Pi_{2}^{i}\right.
$$


TABLE III. Anharmonicity and time duration of segments for the $\mathrm{GP}_{2}$ in the multisegment scheme. The data of $\alpha_{1}$ and $\alpha_{2}$ are divided by $2 \pi$.

\begin{tabular}{|c|c|c|c|c|c|c|c|c|c|}
\hline Index & $\alpha_{1}(\mathrm{MHz})$ & $\alpha_{2}(\mathrm{MHz})$ & $t_{1}(\mathrm{~ns})$ & $t_{2}(\mathrm{~ns})$ & $t_{3}(\mathrm{~ns})$ & $t_{4}(\mathrm{~ns})$ & $t_{5}(\mathrm{~ns})$ & $t_{6}(\mathrm{~ns})$ & $t_{\text {gate }}(\mathrm{ns})$ \\
\hline 8 & $\infty$ & $\infty$ & 0 & 80 & 160 & 240 & 320 & & 320 \\
\hline 9 & $\infty$ & $\infty$ & 0 & 80 & 160 & 240 & 320 & & 320 \\
\hline 10 & $\infty$ & $\infty$ & 0 & 80 & 160 & 240 & 320 & & 320 \\
\hline 11 & $\infty$ & $\infty$ & 0 & 80 & 160 & 240 & 320 & & 320 \\
\hline 12 & $\infty$ & $\infty$ & 0 & 80 & 160 & 240 & 320 & & 320 \\
\hline 13 & $\infty$ & $\infty$ & 0 & 80 & 160 & 240 & 320 & & 320 \\
\hline 14 & $\infty$ & $\infty$ & 0 & 80 & 160 & 240 & 320 & & 320 \\
\hline 15 & $\infty$ & $\infty$ & 0 & 80 & 160 & 240 & 320 & & 320 \\
\hline 16 & $\infty$ & $\infty$ & 0 & 80 & 160 & 240 & 320 & & 320 \\
\hline 17 & $\infty$ & $\infty$ & 0 & 80 & 160 & 240 & 320 & & 320 \\
\hline 18 & -300 & -300 & 0 & 100 & 200 & 300 & 400 & 500 & 500 \\
\hline 19 & -330 & -330 & 0 & 100 & 200 & 300 & 400 & 500 & 500 \\
\hline 20 & -350 & -350 & 0 & 100 & 200 & 300 & 400 & 500 & 500 \\
\hline 21 & -380 & -380 & 0 & 100 & 200 & 300 & 400 & 500 & 500 \\
\hline 22 & -400 & -400 & 0 & 100 & 200 & 300 & 400 & 500 & 500 \\
\hline 23 & -300 & -300 & 0 & 100.28 & 200.56 & 300.84 & 401.12 & 501.40 & 501.40 \\
\hline 24 & -330 & -330 & 0 & 100.07 & 200.51 & 300.20 & 399.87 & 500.39 & 500.39 \\
\hline 25 & -350 & -350 & 0 & 100.42 & 200.60 & 299.81 & 399.77 & 500.17 & 500.17 \\
\hline 26 & -380 & -380 & 0 & 100.20 & 200.48 & 299.89 & 399.80 & 500.31 & 500.31 \\
\hline 27 & -400 & -400 & 0 & 100.33 & 200.47 & 299.78 & 399.71 & 500.17 & 500.17 \\
\hline
\end{tabular}

$$
\begin{aligned}
& \left.+\frac{g_{i} \Omega_{i}}{2 \Delta_{i}} \sigma_{1 z}^{i}\left(a+a^{\dagger}\right)+\frac{\sqrt{2} \lambda_{i} g_{i} \Omega_{i}}{2\left(\Delta_{i}-\alpha_{i}\right)} \sigma_{2 z}^{i}\left(a+a^{\dagger}\right)+\frac{g_{i}^{2}}{\widetilde{\Delta}_{1}^{i}-\delta} \sigma_{1 z}^{i} a^{\dagger} a+\frac{2 g_{i}^{2}}{\widetilde{\Delta}_{2}^{i}-\delta} \sigma_{2 z}^{i} a^{\dagger} a\right], \\
H_{\mathrm{err}}= & -\frac{1}{2}\left(g_{1} \beta_{1}^{2}+g_{2} \beta_{1}^{1}\right)\left(\sigma_{1+}^{1} \sigma_{1-}^{2}+\sigma_{1-}^{1} \sigma_{1+}^{2}\right)-\frac{1}{2}\left(g_{2} \beta_{2}^{1}+\sqrt{2} g_{1} \beta_{1}^{2}\right)\left(\sigma_{2+}^{1} \sigma_{1-}^{2}+\sigma_{2-}^{1} \sigma_{1+}^{2}\right) \\
& -\frac{1}{2}\left(g_{1} \beta_{2}^{2}+\sqrt{2} g_{2} \beta_{1}^{1}\right)\left(\sigma_{2+}^{2} \sigma_{1-}^{1}+\sigma_{2-}^{2} \sigma_{1+}^{1}\right)-\frac{\sqrt{2}}{2}\left(g_{1} \beta_{2}^{2}+g_{2} \beta_{2}^{1}\right)\left(\sigma_{2+}^{1} \sigma_{2-}^{2}+\sigma_{2-}^{1} \sigma_{2+}^{2}\right) \\
+ & \sum_{i=1,2}\left[\frac{\lambda_{i} \Omega_{i}^{2} \alpha_{i}}{8 \Delta_{i}\left(\alpha_{i}-\Delta_{i}\right)}\left(|2\rangle_{i}\langle 0|+| 0\rangle_{i}\langle 2|\right)+\frac{1}{2}\left(-\frac{\lambda_{i} g_{i} \Omega_{i}}{\Delta_{i}-\alpha_{i}}+\frac{\sqrt{2} g_{i} \Omega_{i}}{\Delta_{i}}\right)\left(|2\rangle_{i}\langle 0|a+| 0\rangle_{i}\langle 2| a^{\dagger}\right)\right. \\
& \left.\quad-\frac{1}{2}\left(g_{i} \beta_{2}^{i}-\sqrt{2} g_{i} \beta_{1}^{i}\right)\left(|2\rangle_{i}\left\langle 0\left|a^{2}+\right| 0\right\rangle_{i}\langle 2| a^{\dagger 2}\right)\right],
\end{aligned}
$$

where $\sigma_{1 z}^{i}=\Pi_{0}^{i}-\Pi_{1}^{i}, \sigma_{2 z}^{i}=\Pi_{1}^{i}-\Pi_{2}^{i}, \beta_{1}^{i}=\frac{g_{i}}{\Delta_{1}^{i}-\delta}, \beta_{2}^{i}=\frac{\sqrt{2} g_{i}}{\Delta_{2}^{i}-\delta}(i=1,2)$. Then, in analogy to Eq. (A12), $U_{\text {gate }}$ can be calculated in the interaction picture as

$$
\begin{aligned}
U_{\text {gate }} & =U_{k}\left(\tau_{k}\right) \cdots U_{j}\left(\tau_{j}\right) \cdots U_{2}\left(\tau_{2}\right) U_{1}\left(\tau_{1}\right), \\
U_{j}(t) & =e^{\mathcal{F}\left(\Omega_{j}, t\right)+i \beta(t)\left(\eta_{1}^{1} \sigma_{1 z}^{1}+\eta_{2}^{1} \sigma_{2 z}^{1}+\eta_{1}^{2} \sigma_{1 z}^{2}+\eta_{2}^{2} \sigma_{2 z}^{2}\right)^{2} t},
\end{aligned}
$$

where $\beta(t)=\frac{1}{\delta_{q d}^{2}}\left(-\delta_{q d}^{\prime} t+\sin \delta_{q d}^{\prime} t\right), \delta_{q d}^{\prime}=\delta-\sum_{i=1,2}\left(\frac{g_{i}^{2}}{\Delta_{i}-\delta} \sigma_{1 z}^{i}+\frac{2 g_{i}^{2}}{\Delta_{i}-\alpha_{i}-\delta} \sigma_{2 z}^{i}\right), \eta_{1}^{i}=\frac{g_{i} \Omega_{i}}{2 \Delta_{i}}, \eta_{2}^{i}=\frac{\sqrt{2} g_{i} \Omega_{i} \lambda_{i}}{2\left(\Delta_{i}+\alpha_{i}\right)}(i=1,2) . \mathcal{F}\left(\Omega_{j}, t\right)$ represents the displacement operation of a photon in the bus. Here, the microwave pulse is supposed to be split into $k$ segments, and the duration time of each pulse is $\tau_{j}(j=1,2, \ldots, k)$.

\section{APPENDIX C: SIMULATION PARAMETERS}

In this Appendix, we present our device parameters used for the simulation shown in the main text. Table I shows the fundamental parameters for realizing the two-qubit geometric phase gate; meanwhile, Tables II and III give the supplementary data to Table I in the multisegment scheme. Table IV displays the simulation parameters for realizing the threequbit geometric phase gate and the many-body interaction.

Indices 1-7 in Table I explore the influence of approximate conditions on $\mathrm{GP}_{2}$ gate fidelity based on the single-segment scheme for a qubit model, and the corresponding results are depicted in Fig. 2(d) in the main text. Indices $8-13$ in Table I verify the gate infidelity caused by the relatively poor approximate conditions in the single-segment scheme for typical transmon parameters. Apparently, the results plotted in Fig. 3(c) in the main text reveal that the $\mathrm{GP}_{2}$ may fail once the frequency detuning between qubits and bus is close. Further optimization with the multisegment scheme $(k=4)$ is adopted to verify the effectiveness of the multisegment method for solving the evolution of the photon. The 
TABLE IV. Simulation parameters for the $\mathrm{GP}_{3}$. All data except for those of $t_{\text {gate }}$ are divided by $2 \pi$. We omit the units for all parameters here. The corresponding units are displayed in Table I.

\begin{tabular}{cccccccccccccccc}
\hline \hline Index & $\omega_{1}$ & $\omega_{2}$ & $\omega_{3}$ & $\omega_{b}$ & $g_{1}$ & $g_{2}$ & $g_{3}$ & $\Omega_{1}$ & $\Omega_{2}$ & $\Omega_{3}$ & $\delta$ & $\Delta_{1}$ & $\Delta_{2}$ & $\Delta_{3}$ & $t_{\text {gate }}$ \\
\hline 28 & 0.1 & 0.3 & 0.5 & 12.0 & 20 & 20 & 20 & 595.2 & 585.2 & 575.2 & 4 & 11904 & 11704 & 11504 & 250 \\
29 & 5.074 & 5.144 & 5.214 & 5.5 & 20 & 20 & 20 & & & & 4 & 430 & 360 & 290 & 900 \\
& $\alpha_{1}$ & $\alpha_{2}$ & $\alpha_{3}$ & $\Omega_{11}$ & $\Omega_{12}$ & $\Omega_{13}$ & $\Omega_{14}$ & $\Omega_{15}$ & $\Omega_{16}$ & $\Omega_{21}$ & $\Omega_{22}$ & $\Omega_{23}$ & $\Omega_{24}$ & $\Omega_{25}$ & $\Omega_{26}$ \\
& -380 & -380 & -380 & -0.01 & -0.43 & -5.12 & -7.93 & -4.57 & -0.09 & -0.54 & -8.85 & -20.71 & -24.55 & -19.57 & -7.47 \\
& $\Omega_{31}$ & $\Omega_{32}$ & $\Omega_{33}$ & $\Omega_{34}$ & $\Omega_{35}$ & $\Omega_{36}$ & $t_{1}$ & $t_{2}$ & $t_{3}$ & $t_{4}$ & $t_{5}$ & $t_{6}$ & $t_{7}$ & \\
& 0.64 & 10.04 & 18.74 & 24.51 & 17.70 & 8.81 & 0 & 150 & 300 & 450 & 600 & 750 & 900 & \\
\hline \hline
\end{tabular}

corresponding pulse parameters are shown in Tables II and III with the improved gate fidelity plotted in Fig. 3(c) in the main text. Indices 8 and 14-17 in Tables I-III explore different settings for the qubit frequencies compared with the bus. The outcomes exhibited in Fig. 3(d) demonstrate that high gate fidelity can be achieved using the multisegment scheme for various frequency setups. Indices 18-22 in Tables I-III demonstrate the effect of the qubit anharmonicity on the gate fidelity based on the multisegment scheme $(k=5)$ under the multilevel model, shown in Fig. 3(f) in the main text. Indices 23-27 exhibit the optimized parameters via the further "parameter-search" method, demonstrating the nearly perfect gate fidelity shown in Fig. 3(f) in the main text. Index 28 in Table IV is an example of simulation parameters for realizing $\mathrm{GP}_{3}$ in the single-segment scheme which is similar to index 7. The parameters of index 29 in Table IV are used to verify the generation of many-body interactions for a transmon, revealing a potential application for controlling phase accumulation during the multiqubit geometric phase gate via the multisegment scheme.
[1] M. V. Berry, Quantal phase factors accompanying adiabatic changes, Proc. R. Soc. London Ser. A 392, 45 (1984).

[2] B. Simon, Holonomy, the Quantum Adiabatic Theorem, and Berry's Phase, Phys. Rev. Lett. 51, 2167 (1983).

[3] F. Wilczek and A. Zee, Appearance of Gauge Structure in Simple Dynamical Systems, Phys. Rev. Lett. 52, 2111 (1984).

[4] Y. Aharonov and J. Anandan, Phase Change during a Cyclic Quantum Evolution, Phys. Rev. Lett. 58, 1593 (1987).

[5] F. Wilczek and A. Shapere, Geometric Phases in Physics (World Scientific, Singapore, 1989).

[6] D. Xiao, M.-C. Chang, and Q. Niu, Berry phase effects on electronic properties, Rev. Mod. Phys. 82, 1959 (2010).

[7] J. Dalibard, F. Gerbier, G. Juzeliūnas, and P. Öhberg, Colloquium: Artificial gauge potentials for neutral atoms, Rev. Mod. Phys. 83, 1523 (2011).

[8] S.-L. Zhu and P. Zanardi, Geometric quantum gates that are robust against stochastic control errors, Phys. Rev. A 72, 020301(R) (2005).

[9] P. Solinas, M. Sassetti, P. Truini, and N. Zanghì, On the stability of quantum holonomic gates, New J. Phys. 14, 093006 (2012).

[10] M. Johansson, E. Sjöqvist, L. M. Andersson, M. Ericsson, B. Hessmo, K. Singh, and D. M. Tong, Robustness of nonadiabatic holonomic gates, Phys. Rev. A 86, 062322 (2012).

[11] S. Berger, M. Pechal, A. A. Abdumalikov, C. Eichler, L. Steffen, A. Fedorov, A. Wallraff, and S. Filipp, Exploring the effect of noise on the Berry phase, Phys. Rev. A 87, 060303(R) (2013).

[12] A. Carollo, I. Fuentes-Guridi, M. F. Santos, and V. Vedral, Geometric Phase in Open Systems, Phys. Rev. Lett. 90, 160402 (2003).

[13] S.-B. Zheng, Geometric phase for a driven quantum field subject to decoherence, Phys. Rev. A 91, 052117 (2015).

[14] P. Zanardi and M. Rasetti, Holonomic quantum computation, Phys. Lett. A 264, 94 (1999).
[15] J. Pachos, P. Zanardi, and M. Rasetti, Non-Abelian Berry connections for quantum computation, Phys. Rev. A 61, 010305(R) (1999).

[16] L.-M. Duan, J. I. Cirac, and P. Zoller, Geometric manipulation of trapped ions for quantum computation, Science 292, 1695 (2001).

[17] E. Sjöqvist, Trend: A new phase in quantum computation, Physics 1, 35 (2008).

[18] J. A. Jones, V. Vedral, A. Ekert, and G. Castagnoli, Geometric quantum computation using nuclear magnetic resonance, Nature (London) 403, 869 (2000).

[19] G. Feng, G. Xu, and G. Long, Experimental Realization of Nonadiabatic Holonomic Quantum Computation, Phys. Rev. Lett. 110, 190501 (2013).

[20] Z. Zhu, T. Chen, X. Yang, J. Bian, Z.-Y. Xue, and X. Peng, Single-Loop and Composite-Loop Realization of Nonadiabatic Holonomic Quantum Gates in a Decoherence-Free Subspace, Phys. Rev. Appl. 12, 024024 (2019).

[21] S. Arroyo-Camejo, A. Lazariev, S. W. Hell, and G. Balasubramanian, Room temperature high-fidelity holonomic single-qubit gate on a solid-state spin, Nat. Commun. 5, 4870 (2014).

[22] C. Zu, W.-B. Wang, L. He, W.-G. Zhang, C.-Y. Dai, F. Wang, and L.-M. Duan, Experimental realization of universal geometric quantum gates with solid-state spins, Nature (London) 514, 72 (2014).

[23] Y. Sekiguchi, N. Niikura, R. Kuroiwa, H. Kano, and H. Kosaka, Optical holonomic single quantum gates with a geometric spin under a zero field, Nat. Photon. 11, 309 (2017).

[24] K. Nagata, K. Kuramitani, Y. Sekiguchi, and H. Kosaka, Universal holonomic quantum gates over geometric spin qubits with polarised microwaves, Nat. Commun. 9, 3277 (2018).

[25] Y.-Y. Huang, Y.-K. Wu, F. Wang, P.-Y. Hou, W.-B. Wang, W.-G. Zhang, W.-Q. Lian, Y.-Q. Liu, H.-Y. Wang, H.-Y. Zhang, L. He, 
X.-Y. Chang, Y. Xu, and L.-M. Duan, Experimental Realization of Robust Geometric Quantum Gates with Solid-State Spins, Phys. Rev. Lett. 122, 010503 (2019).

[26] D. Leibfried, B. DeMarco, V. Meyer, D. Lucas, M. Barrett, J. Britton, W. M. Itano, B. Jelenković, C. Langer, T. Rosenband, and D. J. Wineland, Experimental demonstration of a robust, high-fidelity geometric two ion-qubit phase gate, Nature (London) 422, 412 (2003).

[27] K. Toyoda, K. Uchida, A. Noguchi, S. Haze, and S. Urabe, Realization of holonomic single-qubit operations, Phys. Rev. A 87, 052307 (2013).

[28] A. A. Abdumalikov Jr, J. M. Fink, K. Juliusson, M. Pechal, S. Berger, A. Wallraff, and S. Filipp, Experimental realization of non-Abelian non-adiabatic geometric gates, Nature (London) 496, 482 (2013).

[29] Y. Xu, W. Cai, Y. Ma, X. Mu, L. Hu, T. Chen, H. Wang, Y. P. Song, Z.-Y. Xue, Z.-Q. Yin, and L. Sun, Single-Loop Realization of Arbitrary Nonadiabatic Holonomic Single-Qubit Quantum Gates in a Superconducting Circuit, Phys. Rev. Lett. 121, 110501 (2018).

[30] T. Yan, B.-J. Liu, K. Xu, C. Song, S. Liu, Z. Zhang, H. Deng, Z. Yan, H. Rong, K. Huang, M.-H. Yung, Y. Chen, and D. Yu, Experimental Realization of Nonadiabatic Shortcut to NonAbelian Geometric Gates, Phys. Rev. Lett. 122, 080501 (2019).

[31] D. J. Egger, M. Ganzhorn, G. Salis, A. Fuhrer, P. Müller, P. K. Barkoutsos, N. Moll, I. Tavernelli, and S. Filipp, Entanglement Generation in Superconducting Qubits Using Holonomic Operations, Phys. Rev. Appl. 11, 014017 (2019).

[32] Y. Xu, Z. Hua, T. Chen, X. Pan, X. Li, J. Han, W. Cai, Y. Ma, H. Wang, Y. P. Song, Z.-Y. Xue, and L. Sun, Experimental Implementation of Universal Nonadiabatic Geometric Quantum Gates in a Superconducting Circuit, Phys. Rev. Lett. 124, 230503 (2020).

[33] C. Song, S.-B. Zheng, P. Zhang, K. Xu, L. Zhang, Q. Guo, W. Liu, D. Xu, H. Deng, K. Huang, D. Zheng, X. Zhu, and H. Wang, Continuous-variable geometric phase and its manipulation for quantum computation in a superconducting circuit, Nat. Commun. 8, 1061 (2017).

[34] K. Xu, W. Ning, X.-J. Huang, P.-R. Han, H. Li, Z.-B. Yang, D. Zheng, H. Fan, and S.-B. Zheng, Demonstration of a nonAbelian geometric controlled-NOT gate in a superconducting circuit, Optica 8, 972 (2021).

[35] X.-B. Wang and M. Keiji, Nonadiabatic Conditional Geometric Phase Shift with NMR, Phys. Rev. Lett. 87, 097901 (2001); 88, 179901(E) (2002).

[36] S.-L. Zhu and Z. D. Wang, Implementation of Universal Quantum Gates Based on Nonadiabatic Geometric Phases, Phys. Rev. Lett. 89, 097902 (2002).

[37] E. Sjöqvist, D. M. Tong, L. M. Andersson, B. Hessmo, M. Johansson, and K. Singh, Non-adiabatic holonomic quantum computation, New J. Phys. 14, 103035 (2012).

[38] G. F. Xu, J. Zhang, D. M. Tong, E. Sjöqvist, and L. C. Kwek, Nonadiabatic Holonomic Quantum Computation in Decoherence-Free Subspaces, Phys. Rev. Lett. 109, 170501 (2012).

[39] P. J. Leek, J. M. Fink, A. Blais, R. Bianchetti, M. Göppl, J. M. Gambetta, D. I. Schuster, L. Frunzio, R. J. Schoelkopf, and A. Wallraff, Observation of Berry's phase in a solid-state qubit, Science 318, 1889 (2007).
[40] S.-L. Zhu and Z. D. Wang, Unconventional Geometric Quantum Computation, Phys. Rev. Lett. 91, 187902 (2003).

[41] A. Sørensen and K. Mølmer, Quantum Computation with Ions in Thermal Motion, Phys. Rev. Lett. 82, 1971 (1999).

[42] G. Milburn, S. Schneider, and D. James, Ion trap quantum computing with warm ions, Fortschr. Phys. 48, 801 (2000).

[43] A. Sørensen and K. Mølmer, Entanglement and quantum computation with ions in thermal motion, Phys. Rev. A 62, 022311 (2000).

[44] C. J. Ballance, T. P. Harty, N. M. Linke, M. A. Sepiol, and D. M. Lucas, High-Fidelity Quantum Logic Gates Using Trapped-Ion Hyperfine Qubits, Phys. Rev. Lett. 117, 060504 (2016).

[45] J. P. Gaebler, T. R. Tan, Y. Lin, Y. Wan, R. Bowler, A. C. Keith, S. Glancy, K. Coakley, E. Knill, D. Leibfried, and D. J. Wineland, High-Fidelity Universal Gate Set for ${ }^{9} \mathrm{Be}^{+}$Ion Qubits, Phys. Rev. Lett. 117, 060505 (2016).

[46] S.-L. Zhu, Z. D. Wang, and P. Zanardi, Geometric Quantum Computation and Multiqubit Entanglement with Superconducting Qubits inside a Cavity, Phys. Rev. Lett. 94, 100502 (2005).

[47] Z.-Y. Xue and Z. D. Wang, Simple unconventional geometric scenario of one-way quantum computation with superconducting qubits inside a cavity, Phys. Rev. A 75, 064303 (2007).

[48] B. Royer, A. L. Grimsmo, N. Didier, and A. Blais, Fast and high-fidelity entangling gate through parametrically modulated longitudinal coupling, Quantum 1, 11 (2017).

[49] A. J. Kerman, Quantum information processing using quasiclassical electromagnetic interactions between qubits and electrical resonators, New J. Phys. 15, 123011 (2013).

[50] S. Touzard, A. Kou, N. E. Frattini, V. V. Sivak, S. Puri, A. Grimm, L. Frunzio, S. Shankar, and M. H. Devoret, Gated Conditional Displacement Readout of Superconducting Qubits, Phys. Rev. Lett. 122, 080502 (2019).

[51] M. Pechal, S. Berger, A. A. Abdumalikov, J. M. Fink, J. A. Mlynek, L. Steffen, A. Wallraff, and S. Filipp, Geometric Phase and Nonadiabatic Effects in an Electronic Harmonic Oscillator, Phys. Rev. Lett. 108, 170401 (2012).

[52] S.-L. Zhu, C. Monroe, and L.-M. Duan, Arbitrary-speed quantum gates within large ion crystals through minimum control of laser beams, Europhys. Lett. 73, 485 (2006).

[53] S. Sheldon, E. Magesan, J. M. Chow, and J. M. Gambetta, Procedure for systematically tuning up cross-talk in the crossresonance gate, Phys. Rev. A 93, 060302(R) (2016).

[54] A. W. Cross and J. M. Gambetta, Optimized pulse shapes for a resonator-induced phase gate, Phys. Rev. A 91, 032325 (2015).

[55] H. Paik, A. Mezzacapo, M. Sandberg, D. T. McClure, B. Abdo, A. D. Córcoles, O. Dial, D. F. Bogorin, B. L. T. Plourde, M Steffen, A. W. Cross, J. M. Gambetta, and J. M. Chow, Experimental Demonstration of a Resonator-Induced Phase Gate in a Multiqubit Circuit-QED System, Phys. Rev. Lett. 117, 250502 (2016).

[56] V. E. Manucharyan, J. Koch, L. I. Glazman, and M. H. Devoret, Fluxonium: Single Cooper-pair circuit free of charge offsets, Science 326, 113 (2009).

[57] L. B. Nguyen, Y.-H. Lin, A. Somoroff, R. Mencia, N. Grabon, and V. E. Manucharyan, High-Coherence Fluxonium Qubit, Phys. Rev. X 9, 041041 (2019). 
[58] N. Goldman and J. Dalibard, Periodically Driven Quantum Systems: Effective Hamiltonians And Engineered Gauge Fields, Phys. Rev. X 4, 031027 (2014).

[59] J. Johansson, P. Nation, and F. Nori, QuTiP 2: A Python framework for the dynamics of open quantum systems, Comput. Phys. Commun. 184, 1234 (2013).

[60] J. Koch, T. M. Yu, J. Gambetta, A. A. Houck, D. I. Schuster, J. Majer, A. Blais, M. H. Devoret, S. M. Girvin, and R. J. Schoelkopf, Charge-insensitive qubit design derived from the Cooper pair box, Phys. Rev. A 76, 042319 (2007).

[61] J. M. Gambetta, F. Motzoi, S. T. Merkel, and F. K. Wilhelm, Analytic control methods for high-fidelity unitary operations in a weakly nonlinear oscillator, Phys. Rev. A 83, 012308 (2011).

[62] P. Virtanen, R. Gommers, T. E. Oliphant, M. Haberland, T. Reddy, D. Cournapeau, E. Burovski, P. Peterson, W. Weckesser, J. Bright, S. J. van der Walt, M. Brett, J. Wilson, K. J.
Millman, N. Mayorov, A. R. J. Nelson, E. Jones, R. Kern, E. Larson, C. J. Carey et al., SciPy 1.0: fundamental algorithms for scientific computing in Python, Nat. Methods 17, 261 (2020).

[63] F. Yan, P. Krantz, Y. Sung, M. Kjaergaard, D. L. Campbell, T. P. Orlando, S. Gustavsson, and W. D. Oliver, Tunable Coupling Scheme for Implementing High-Fidelity Two-Qubit Gates, Phys. Rev. Appl. 10, 054062 (2018).

[64] X. Li, T. Cai, H. Yan, Z. Wang, X. Pan, Y. Ma, W. Cai, J. Han, Z. Hua, X. Han, Y. Wu, H. Zhang, H. Wang, Y. Song, L. Duan, and L. Sun, Tunable Coupler for Realizing a Controlled-Phase Gate with Dynamically Decoupled Regime in a Superconducting Circuit, Phys. Rev. Appl. 14, 024070 (2020).

[65] S. Bravyi, D. P. DiVincenzo, and D. Loss, Schrieffer-Wolff transformation for quantum many-body systems, Ann. Phys. (Amsterdam) 326, 2793 (2011). 